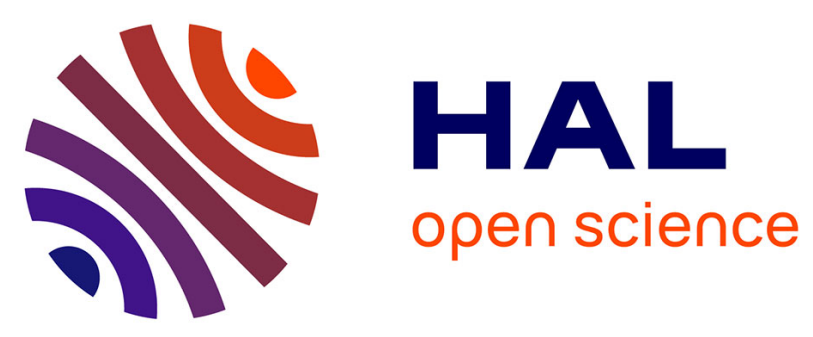

\title{
De l'importance du maintien de la préférence communautaire pour l'agriculture française. Une illustration à l'aide du modèle MEGAAF
}

\author{
Alexandre Gohin, Herve Guyomard, Chantal Le Mouël
}

\section{- To cite this version:}

Alexandre Gohin, Herve Guyomard, Chantal Le Mouël. De l'importance du maintien de la préférence communautaire pour l'agriculture française. Une illustration à l'aide du modèle MEGAAF. Journée INRA-SFER "L'agriculture dans l'économie: modélisations macro-économiques, modèles d'équilibre général calculable", Apr 1998, Paris, France. 28 p., 1998. hal-02837882

\section{HAL Id: hal-02837882 \\ https://hal.inrae.fr/hal-02837882}

Submitted on 7 Jun 2020

HAL is a multi-disciplinary open access archive for the deposit and dissemination of scientific research documents, whether they are published or not. The documents may come from teaching and research institutions in France or abroad, or from public or private research centers.
L'archive ouverte pluridisciplinaire HAL, est destinée au dépôt et à la diffusion de documents scientifiques de niveau recherche, publiés ou non, émanant des établissements d'enseignement et de recherche français ou étrangers, des laboratoires publics ou privés.

\section{(1) (1) $\$$}

Distributed under a Creative Commons Attribution - NonCommercial - NoDerivatives 44.0 
L'AGRICULTURE DANS L'ECONOMIE :

modélisations macro-économiques, modèles d'équilibre général calculable.

JOURNEE INRA-SFER

PARIS 29 Avril 1998

\title{
De l'importance du maintien de la préférence communautaire pour le futur des secteurs agricole et agro-alimentaire français : Une illustration à l'aide du modèle MEGAAF
}

\author{
A. Gohin, H. Guyomard, C. Le Mouël \\ Equipe PAM, Unité INRA-ESR, Rennes
}

\section{RESUME :}

L'objectif de ce papier est de mettre en évidence l'importance du maintien de la préférence communautaire au regard du futur des secteurs agricole et agro-alimentaire français, dans la perspective d'évolution de la PAC vers l'affaiblissement du rôle des systèmes de soutien par les prix au profit d'autres formes de soutien à caractère plus découplé. Pour ce faire, deux scénarios de réforme radicale de l'OCM des céréales et des oléoprotéagineux (COP), impliquant la suppression des mesures de soutien interne avec ou sans maintien de la protection tarifaire vis-à-vis des importations étrangères, sont simulés à l'aide du modèle MEGAAF. A cet égard, nous proposons une modélisation du bloc des importations françaises de céréales en provenance des pays tiers qui, contrairement à la modélisation de type Armington généralement adoptée dans les modèles d'EGC, permet de rendre compte de manière endogène de changements de régime d'importation, i.e. de représenter un réel mouvement de pénétration des importations céréalières étrangères sur les marchés français, suite à l'abandon de ia protection tarifaire. Les résultats de simulations montrent clairement que la suppression des mesures de soutien interne dans le secteur des COP induit un choc en termes de contraction de l'activité de ce secteur beaucoup plus limité lorsque la préférence communautaire est maintenue que lorsque cette dernière est simultanément supprimée. Ils suggèrent en outre que l'abandon de la préférence communautaire porte le risque de remettre en cause l'indépendance de la France (et plus généralement de l'UE) vis-à-vis des importations céréalières étrangères. 
L'AGRICULTURE DANS L'ECONOMIE :

modélisations macro-économiques, modèles d'équilibre général calculable.

JOURNEE INRA-SFER

\title{
De l'importance du maintien de la préférence communautaire pour le futur des secteurs agricole et agro-alimentaire français : Une illustration à l'aide du modèle MEGAAF
}

\author{
A. Gohin, H. Guyomard, C. Le Mouël \\ Equipe PAM, Unité INRA-ESR, Rennes
}

\section{Introduction}

L'importance de l'Accord Agricole de l'Uruguay Round (AAUR), signé à Marrakech en avril 1994, ne tient pas tant à ses effets quantitatifs immédiats sur les marchés agricoles mais à ses conséquences au regard de l'évolution future des politiques agricoles des pays membres de l'Organisation Mondiale du Commerce (OMC). Cet Accord représente un pas significatif pour soumettre l'agriculture à la discipline générale de l'OMC. Il marque en effet la fin d'une période où les politiques agricoles étaient, à l'exception de quelques concessions ponctuelles, élaborées indépendamment de l'OMC.

Le prochain cycle de négociations commerciales multilatérales, qui doit démarrer en 1999, ouvrira à nouveau le dossier agricole. A cet égard, l'instrumentation du cycle Uruguay constituera la base de la négociation. Il est donc vraisemblable que l'objectif des pays favorables à une plus grande libéralisation des marchés, Etats-Unis et Groupe de Cairns en particulier, sera de poursuivre le mouvement déjà engagé de réduction des exportations subventionnées, d'accroissement des possibilités d'accès au marché et de diminution des niveaux de soutien interne. En outre, il est très probable que plusieurs parties contractantes, Etats-Unis en tête, chercheront également à discipliner les politiques de soutien interne en renforçant l'orientation en faveur des aides directes découplées, i.e. ayant une influence réduite sur le comportement d'offre du producteur et indépendantes des conditions de marché, au détriment des instruments plus couplés, tels que les systèmes de soutien par les prix'.

\footnotetext{
${ }^{1}$ Il est vraisemblable que lors du prochain cycle de négociations de l'OMC, la définition des instruments de soutien interne autorisés et leur classification dans les différentes "boîtes" sera un élément important des débats. A cet égard, la nouvelle loi agricole américaine ("FAIR Act"), adoptée le 5 avril 1996, préfigure la future position de négociation des Etats-Unis au sein de l'OMC. Ces derniers argueront sans nul doute du plus grand découplage de leurs nouvelles aides directes (les "Production Flexibility Contract Payments") et de leur probable classement dans la "boîte verte" pour remettre en cause l'exception de la
} 
Comme toutes les politiques agricoles des pays membres de l'OMC, la Politique Agricole Commune (PAC) évoluera donc dans le contexte et sous la contrainte des négociations internationales. Cette contrainte a déjà pesé sur l'évolution de la PAC puisqu'il est à présent généralement admis que la réforme de mai 1992 répondait au souci de l'Union Européenne (UE) de parvenir à un accord dans le cadre du cycle de négociations Uruguay, de façon à satisfaire ses partenaires commerciaux et ainsi à affaiblir leurs attaques répétées contre la PAC (Guyomard et Mahé, 1995a, Gohin et al., 1998a). La contrainte de l'OMC continue d'être active aujourd'hui, comme en témoigne le contenu de la proposition de réforme de la PAC présentée par la Commission Européenne en juillet 1997 dans son "Agenda 2000" (proposition également connue sous le terme de "paquet Santer"). Les mesures proposées, qui correspondent globalement à un nouveau pas dans la direction suivie en mai 1992, sont en effet clairement destinées à satisfaire à la contrainte de réduction des exportations subventionnées, actuelle i.e. issue de l'AAUR, et anticipée, i.e. qui résultera du prochain cycle de négociations à l'OMC (Guyomard, 1997). Enfin, il est clair que la contrainte de l'OMC qui se profile à l'horizon des prochaines négociations internationales conditionnera l'évolution de la PAC, au travers à la fois du renforcement des engagements sur les trois volets, i.e. concurrence à l'exportation, accès au marché et soutien interne, et de la définition plus sévère des critères de découplage qui forcera l'UE à revoir son système actuel de soutien au revenu des producteurs, et en particulier à redéfinir les modalités d'attribution des aides compensatoires de la réforme de façon à ce qu'elles soient, sans ambiguïté, classées dans la "boîte verte"2.

Ainsi, il semble que la PAC, sous l'effet des contraintes issues des négociations internationales ${ }^{3}$, soit engagée dans un mouvement de réduction graduelle du soutien par les prix, ce dernier étant remplacé par d'autres formes de soutien plus découplé. Les ajustements de la PAC, engagés dans le cadre de la réforme de mai 1992 et proposés par la Commission Européenne dans son "Agenda 2000", s'inscrivent parfaitement dans ce mouvement puisqu'ils consistent essentiellement à réduire les prix de soutien en vigueur dans les principales Organisations Communes de Marché (OCM), ces baisses de prix étant compensées par des aides directes, partiellement découplées, aux producteurs. En contribuant à diminuer le niveau des restitutions à l'exportation requises pour exporter sur pays tiers, de tels ajustements de la PAC constituent une réponse à la contrainte de l'AAUR reconnue comme la plus pressante, i.e. celle relevant des engagements de réduction, en volume et en valeur, des exportations subventionnées ${ }^{4}$. Ils permettent également, par la même occasion, de réduire le niveau de soutien interne observé dans l'UE, desserrant ainsi la contrainte résultant des engagements, passé et futur, en matière de diminution du soutien interne.

"boîte bleue", i.e. l'exemption des paiements compensatoires de la réforme de la PAC de l'engagement de réduction du soutien interne. Pour plus de détails sur ce point, voir Bureau et al. (1996).

${ }^{2}$ Il est aujourd'hui largement admis que les paiements compensatoires de la réforme, assis sur la surface dans le cas des céréales et des oléo-protéagineux (COP) et sur le cheptel en ce qui concerne la production bovine, ne sont que partiellement découplés (voir par exemple Guyomard et Mahé, 1995b, Guyomard et al., 1996a).

${ }^{3}$ Soulignons ici que de nombreuses autres contraintes pèsent sur l'évolution de la PAC : élargissement de l'UE aux Pays d'Europe Centrale et Orientale (PECO), pression budgétaire interne, meilleure prise en compte des effets externes négatifs et positifs de l'activité agricole dans une perspective de développement durable, pression accrue des consommateurs pour la définition d'une PAC qui reconnaisse leurs intérêts et leurs préoccupations, etc.

${ }^{4}$ Voir par exemple, Josling et Tangermann (1992), Josling (1993), Helmar et al. (1994), USDA (1994), Guyomard et al. (1996b). 
Jusqu'à présent, ces ajustements de la PAC ont été engagés ou sont proposés en considérant comme acquis le maintien de la préférence communautaire, i.e. le maintien d'un niveau de protection des marchés agricoles communautaires suffisant vis-à-vis de la concurrence des importations étrangères. Il est vrai que les équivalents tarifaires de base, consolidés par l'UE à l'OMC dans le cadre des engagements sur le volet de l'accès au marché de l'AAUR, sont suffisamment élevés pour qu'en dépit de leur réduction requise de $36 \%$, la préférence communautaire continue d'être assurée jusqu'en 2000, pour la majorité des produits agricoles. Ceci explique sans doute pourquoi les conséquences, induites par les engagements, passés et futurs, en matière de tarification ont été largement négligées dans les débats sur les perspectives d'évolution de la PAC.

Pourtant, et c'est là un élément essentiel au regard du futur de la PAC, le fait que les équivalents tarifaires de l'UE permettent, en pratique, d'assurer la préférence communautaire à court terme ne doit pas masquer qu'à plus long terme, le principe même de la tarification constitue une remise en cause du principe de la préférence communautaire. En effet, alors que les anciens prélèvements variables à l'importation de la PAC assuraient une préférence communautaire absolue en permettant de maintenir des prix minimum fixes (i.e. indépendants des fluctuations des cours mondiaux en écus) à l'importation, les nouveaux équivalents tarifaires n'assurent eux qu'une préférence communautaire relative puisqu'ils laissent les prix minimum à l'importation varier en fonction de l'évolution des cours mondiaux ${ }^{5}$. Ces équivalents tarifaires étant en outre appelés à être réduits au cours des prochains cycles de négociations de l'OMC, la protection des marchés agricoles de l'UE vis-à-vis de la concurrence des importations étrangères, et par là-même, la préférence communautaire pourraient à terme ne plus être assurées.

Dans ce cas, les enjeux pour le secteur agricole communautaire, d'ajustements de la PAC dans le sens d'un remplacement progressif du système de soutien par les prix par des aides directes à caractère découplé, pourraient être très différents de ceux qui ressortent des analyses usuelles de ce type de réforme de la PAC, qui pour la plupart considèrent le maintien de la préférence communautaire comme acquis. L'objectif de ce papier est d'attirer l'attention sur ce point. Plus précisément, il s'agit de montrer que dans la perspective du mouvement général de réduction graduelle des prix de soutien que les négociations internationales contribuent à imposer à la PAC, le maintien d'un certain degré de préférence communautaire est un élément clé au regard du futur du secteur agricole de l'UE.

Pour illustrer cette idée, deux scénarios d'évolution de la PAC dans le secteur des grandes cultures sont simulés à l'aide du Modèle d'Equilibre Général de l'Agriculture et de l'Agro-alimentaire Français (MEGAAF) ${ }^{6}$ : i) suppression des mesures de soutien interne avec maintien de la préférence communautaire (scénario "de référence") et ii) suppression des mesures de soutien interne et abandon de la préférence communautaire (scénario "protection zéro"). Soulignons dès à présent que notre analyse est illustrative et en aucun cas

\footnotetext{
${ }^{5}$ Notons toutefois que l'AAUR prévoit une clause de sauvegarde permettant aux pays signataires de se prémunir contre de fortes fluctuations à la baisse des cours mondiaux. Cette clause de sauvegarde (qui pour l'UE concerne la majorité des produits sous $\mathrm{OCM}$ ) est déclenchée lorsque le prix CAF à l'importation chute de plus de $10 \%$ en dessous du prix mondial de référence correspondant. Dans ce cas, un droit de douane additionnel (plafonné à un tiers de l'équivalent tarifaire initial) peut être appliqué aux importations du produit considéré.

${ }^{6} \mathrm{Par}$ voie de conséquence, notre analyse ne considère pas l'UE dans son ensemble mais est limitée à la France.
} 
prévisionnelle, les scénarios proposés étant très éloignés des débats actuels sur les voies d'évolution envisageables de la PAC. D'une part, on considère ici non pas une réduction graduelle des prix de soutien en vigueur dans l'OCM des céréales et des oléoprotéagineux (COP), mais une suppression de ces prix. En outre, cet abandon du soutien par les prix dans le secteur des COP ne s'accompagne d'aucune compensation sous la forme de systèmes d'aides directes. Enfin, la suppression des barrières tarifaires à l'entrée pour les céréales relève d'une vision à très long terme, les engagements communautaires de l'AAUR en matière de tarification pour ces produits laissant à l'UE une marge de manoeuvre suffisamment large, même si les équivalents tarifaires consolidés devaient être substantiellement réduits lors des prochaines négociations multilatérales à l'OMC?

Le plan du papier est le suivant. La section 2 présente brièvement la structure générale et les principales caractéristiques du modèle MEGAAF. Puis, les deux scénarios envisagés sont décrits de manière détaillée dans la section 3. La section 4 est consacrée à l'analyse des résultats de simulations. Enfin, la section 5 conclut.

\section{MEGAAF : Structure et caractéristiques générales}

MEGAAF est un modèle d'équilibre général calculable (EGC) statique (relevant de l'approche de Shoven et Whalley, 1984), calibré sur les données de l'année 1990, ces dernières étant organisées sous la forme d'une matrice de comptabilité sociale (MCS) de l'économie française. Ce modèle est décrit de manière détaillée dans Gohin (1998). Nous n'en présentons ici que les principales caractéristiques, en portant toutefois une attention particulière au secteur des COP qui est au coeur des scénarios de réforme de la PAC envisagés dans cette analyse.

\subsection{La dimension du modèle}

L'économie française est découpée en 22 secteurs d'activité et 30 produits, dont 9 secteurs d'activité agricoles, produisant 14 biens agricoles distincts, et 6 secteurs d'activité agro-alimentaires produisant 11 biens agroalimentaires distincts. Certains secteurs d'activité sont par conséquent multi-produits. C'est le cas, en particulier, du secteur des grandes cultures qui offre six biens «élémentaires » (le blé tendre, l'orge, le maïs grain, les graines oléagineuses, les protéagineux, les autres céréales). Le tableau 1 ci-dessous rapporte la ventilation en secteurs d'activité et produits de MEGAAF.

\footnotetext{
${ }^{7}$ Les équivalents tarifaires de base consolidés par l'UE lors de l'AAUR s'élèvent à 149 écus par tonne pour le blé tendre, 145 écus par tonne pour l'orge et 147 écus par tonne pour le maïs. Ils doivent être réduits de $36 \%$ entre 1995/96 et 2000/01. Ainsi, en 2000/01, les équivalents tarifaires autorisés pour les trois céréales précédentes s'établissent respectivement à 95,93 et 94 écus par tonne. Toutefois, suite à l'Accord de Blair House entre les Etats-Unis et l'UE, une disposition spécifique d'écrêtement des équivalents tarifaires relatifs aux céréales stipule que le prix minimum à l'entrée sur le territoire communautaire, droit compris, ne peut être supérieur à $155 \%$ du prix d'intervention. Il faudrait par conséquent que les prix mondiaux des céréales s'établissent à des niveaux très bas (38 écus par tonne en fin de période d'application de l'AAUR pour le blé tendre par exemple, selon BIMA, $n^{\circ} 1418$, Février 1994) pour que la protection du marché intérieur communautaire ne soit plus assurée. En outre, dans le cas de cours mondiaux céréaliers très déprimés, un reliquat de protection serait assuré via le droit additionnel prévu par la clause de sauvegarde.
} 
Tableau 1. La désagrégation en secteurs d'activité et en produits retenue dans MEGAAF

\begin{tabular}{l|l}
\hline Secteurs d'activité & Produits \\
\hline a. Agriculture & $\mathbf{1 4}$ \\
\hline Grandes cultures & Blé tendre, orge, maïs grain, oléagineux, protéagineux, autres céréales \\
Viticulture & Vins \\
Autres cultures & Autres produits de cultures \\
Elevage laitier spécialisé & Lait, fourrages, bovins \\
Elevage bovin spécialisé & Bovins, fourrages \\
Elevage mixte bovins-lait & Lait, fourrages, bovins \\
Elevage porcin & Porcs \\
Elevage avicole & Volailles et œufs \\
Autres élevages & Autres produits animaux, fourrages \\
\hline b. Agro-alimentaire & $\mathbf{1 1}$ \\
\hline Industrie des viandes et conserves & Viande bovine, viande porcine, viande avicole, autres viandes \\
Industrie laitière & Agrégat beurre - poudre de lait écrémé, autres produits laitiers \\
Industrie de l'alimentation animale & Aliments composés \\
Industrie de la transformation des céréales & Produits transformés des céréales \\
Industrie des corps gras & Huiles, tourteaux d'oléagineux \\
Autres industries agro-alimentaires & Autres produits agro-alimentaires \\
\hline c. Reste de l'économie & $\mathbf{5}$ \\
\hline Pêche & Pêche \\
Industrie chimique de base & Produits de l'industrie chimique de base \\
Industrie de la parachimie & Produits de la parachimie \\
Autres industries & Autres biens secondaires \\
Services & Services \\
Distribution alimentaire & \\
Distribution non alimentaire & \\
\hline &
\end{tabular}

Cette désagrégation en secteurs d'activité et produits permet alors d'apprécier les impacts de divers scénarios de politique agricole en tenant compte de tous les stades de la chaîne alimentaire et des principales interactions entre cette chaîne alimentaire et le reste de l'économie française.

Une des particularité du modèle est de distinguer deux zones d'échange : l'Union Européenne (UE) et le Reste du Monde (RdM) hors UE. Cette distinction est nécessaire pour tenir compte de l'appartenance de la France à l'UE et du fait que la politique agricole française est « essentiellement » une politique européenne.

\subsection{La production}

Comme dans la majorité des modèles d'EGC, les producteurs maximisent leur profit sous leur contrainte technologique et déterminent les demandes dérivées des trois facteurs primaires de production (travail, capital et terre) en fonction des prix relatifs. En règle générale, les technologies de production sont mono-produit et à rendements d'échelle constant ; les consommations intermédiaires sont déterminées à l'aide de coefficients techniques fixes sous l'hypothèse d'une technologie de production Leontief par rapport aux consommations intermédiaires; les facteurs primaires de production sont combinés à partir de fonction de substitution de type CES (Constant Elasticity of Substitution).

Cependant, pour les secteurs d'activité agricoles, une telle spécification n'est pas adéquate car elle ne rend pas compte des nombreuses possibilités de substitution entre les différentes consommations intermédiaires d'une part, entre les différentes consommations intermédiaires et les différents facteurs primaires de production d'autre part, qui caractérisent la production agricole. Aussi, pour les secteurs d'activité agricoles, les demandes dérivées d'inputs intermédiaires sont modélisées à partir de fonctions de substitution de type CES. Les technologies de production sont également adaptées pour tenir compte de la multi-production de certains secteurs d'activité. A 
cet égard, l'arbre de production du secteur d'activité des grandes cultures est représenté par le graphique 1. Pour simplifier la présentation graphique, nous avons réduit le nombre de biens élémentaires produits par ce secteur d'activité à deux : le blé tendre et le maïs grain. Au niveau 0 de l'arbre de production, le travail et le capital sont combinés selon une fonction CES pour former un agrégat composite travail-capital. Ce dernier est ensuite combiné avec les différents inputs intermédiaires (autres que les semences, les engrais et les produits de traitement des cultures) sur la base de coefficients techniques fixes pour former, au niveau 1 de l'arbre de production, un nouvel agrégat composite travail-capital-inputs intermédiaires. Ce dernier est ensuite réparti entre les différents biens produits (le blé tendre et le maïs grain sur le graphique 1) sur la base d'une fonction de transformation de type CET (Constant Elasticity of Transformation) au niveau 2 de l'arbre de production. Au niveau 3 de l'arbre de production, l'input composite travail-capital-inputs intermédiaires alloué à la culture d'un produit est combiné, sur la base d'une technologie Leontief à coefficients techniques fixes, aux semences de la culture considérée et à un autre input composite terre allouée à la culture considérée-engrais consacrés à la culture considérée-produits de traitement des cultures consacrés à la culture considérée. Cet autre input composite est défini sur la base d'une fonction CES des inputs élémentaires qui le constituent.

\section{Graphique 1. Représentation de la technologie de production dans le secteur d'activité des grandes cultures}

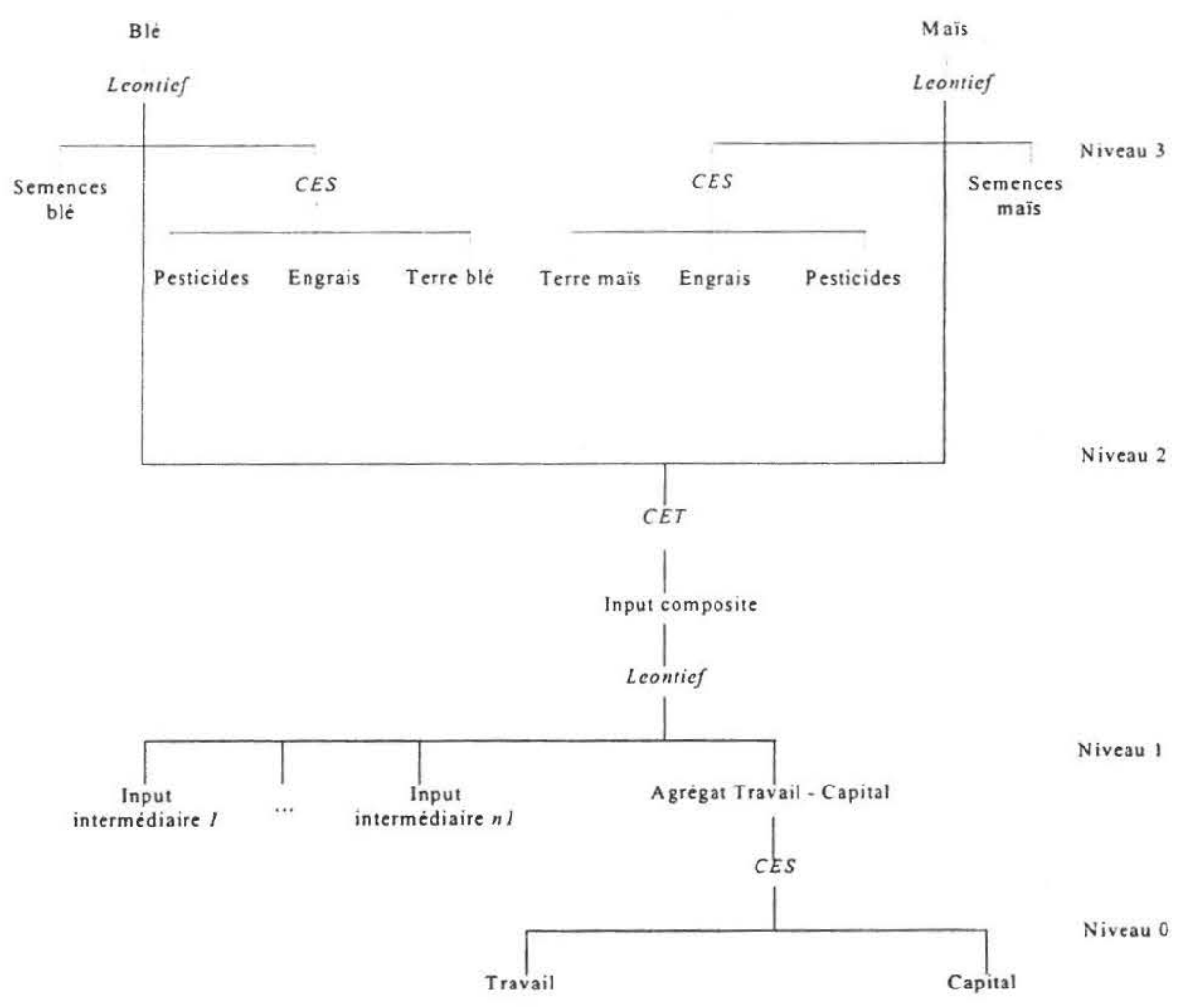

Les trois facteurs primaires de production, dont les quantités offertes sont supposées fixes, sont pleinement utilisés par les 22 secteurs d'activité. Le facteur terre est utilisé uniquement par les secteurs d'activité agricoles et est supposé imparfaitement mobile entre l'ensemble des secteurs animaux et l'ensemble des secteurs végétaux. Cette imparfaite mobilité, modélisée par le biais d'une fonction de transformation de type CET, 
implique que la rémunération unitaire de la terre diffère selon les secteurs agricoles. Les facteurs travail et capital sont utilisés par tous les secteurs d'activité et sont, dans cette analyse, supposés imparfaitement mobiles entre l'ensemble des secteurs d'activité agricoles, l'ensemble des secteurs d'activité agro-alimentaires et l'ensemble des autres secteurs d'activité. En revanche, le travail et le capital sont supposés parfaitement mobiles entre les secteurs d'activité appartenant à un même ensemble.

\subsection{La demande finale}

Les ménages français, représentés par un seul ménage, maximisent une fonction d'utilité sous une contrainte budgétaire. Cette dernière correspond à leur revenu disponible qui provient essentiellement de la rémunération des facteurs primaires de production et des transferts nets avec le gouvernement français. Ce revenu disponible est en partie épargné, le reste étant alloué à la consommation finale des différents biens. Les ménages déterminent donc leur panier de consommation en fonction des prix relatifs des différents biens et de leur revenu disponible. Plus précisément, le revenu disponible à la consommation est réparti entre les différents biens finaux selon un système linéaire de dépense.

\subsection{Les échanges}

Le graphique 2 illustre la modélisation des flux d'importation et d'exportation. La production domestique $Y$ peut être vendue sur le marché domestique $Y D$, exportée sur le marché du reste de l'UE $E_{U E}$ ou exportée sur le marché du RdM hors UE $E_{R d M}$, l'arbitrage étant modélisé à partir d'une fonction de transformation de type CET. Les trois destinations possibles de la production domestique sont donc considérées comme des substituts imparfaits, le degré de différenciation dépendant de la valeur du paramètre de transformation de la fonction CET. Les producteurs domestiques maximisent leur recette sous la contrainte de cette fonction CET; ce programme de maximisation détermine les offres de produits vers les trois destinations possibles, i.e. offres d'exportation vers le reste de l'UE et vers le RdM hors UE et offre de produits domestiques vendus sur le marché domestique, ces trois types d'offre dépendant des prix relatifs observés sur les trois marchés $\left(P E_{U E}, P E_{R d M}, P D\right)$.

La demande domestique totale $X C$ est la somme de la demande intermédiaire des secteurs d'activité $C I T$, de la demande finale des secteurs institutionnels $Q D T$ et de la demande d'investissement $I N V$. De manière symétrique au cas des exportations, cette demande domestique totale peut être satisfaite par les produits d'origine domestique $Y D$, par les importations provenant du reste de l'UE $M_{U E}$ ou par les importations provenant du RdM hors UE $M_{R d M}$. Ces trois types de biens sont considérés comme des substituts imparfaits et sont combinés selon une fonction d'agrégation CES. Le programme de minimisation du coût d'approvisionnement en biens détermine les demandes d'importation et de produits domestiques.

Les hypothèses de substitution imparfaite à l'offre et à la demande entre les biens domestiques et les biens étrangers sont d'usage courant dans les modèles d'EGC (cf., par exemple, Robinson et al., 1990 ; de Melo et Tarr, 1992). Ces hypothèses permettent à un pays d'être à la fois importateur et exportateur pour un "même" bien. 


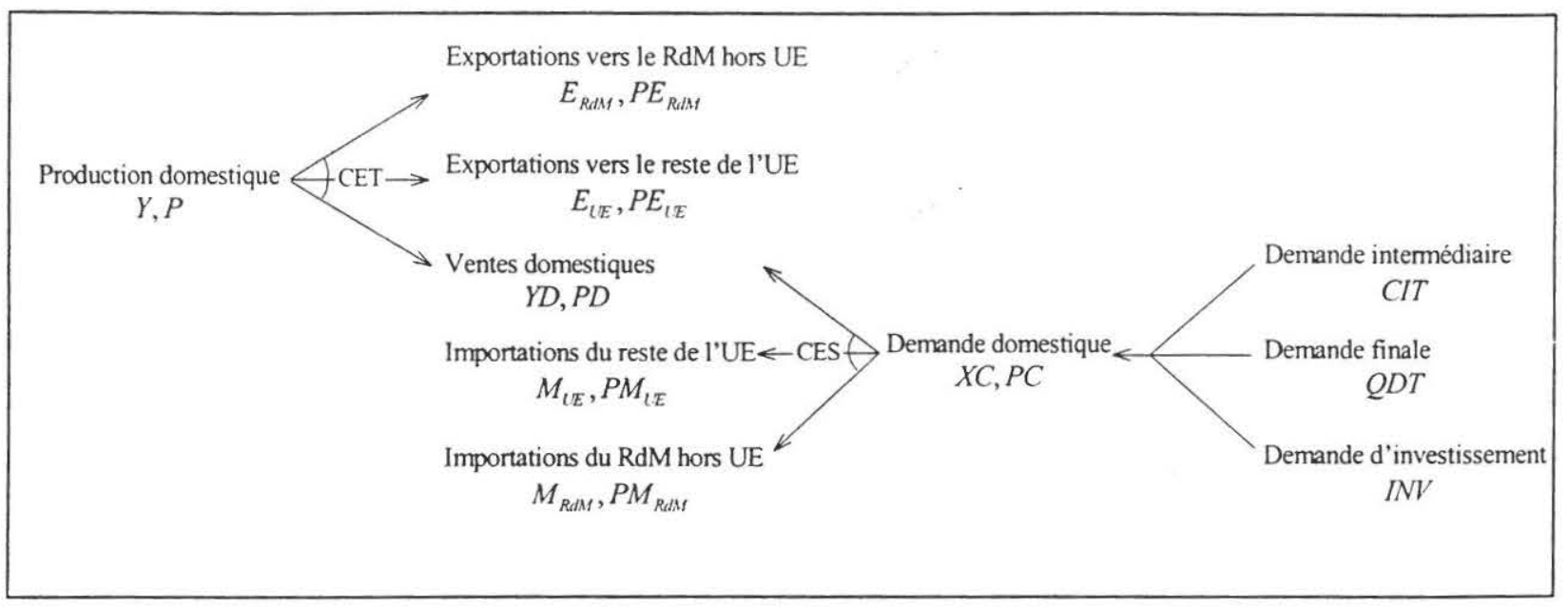

Dans MEGAAF, il est fait l'hypothèse que la France est un "petit pays" à l'importation et à l'exportation sur le marché du RdM hors UE pour tous les produits, excepté les céréales et les produits laitiers. En d'autres termes, les prix mondiaux à l'importation et à l'exportation en devises de tous les produits, hors céréales et produits laitiers, sont des variables exogènes. En revanche, la France est considérée comme un "grand pays" à l'importation et à l'exportation sur le marché du reste de l'UE pour tous les produits, et vis-à-vis du RdM pour les céréales et les produits laitiers. Dans ce cas, les fonctions d'offre d'importation (de demande d'exportation) adressées à la France ne sont pas infiniment élastiques, mais à pente positive (négative).

\subsection{Le bouclage macro-économique}

Le modèle comporte trois composantes macro-économiques. La première correspond à l'équilibre entre l'épargne et l'investissement, la seconde à l'équilibre du budget du gouvernement français, et la troisième à l'équilibre de la balance des paiements. L'investissement est commandé par l'épargne. Le gouvernement français perçoit le produit de toutes les taxes (à la consommation, à la production, ...); il réalise des transferts au profit des ménages français, consomme des services et soutient les secteurs d'activité par des subventions d'exploitation. L'équilibre du budget du gouvernement français peut être assuré via l'ajustement de l'une ou l'autre de ces variables. La règle de bouclage adoptée dans cet exercice stipule que l'équilibre du budget du gouvernement français est assuré par la consommation gouvernementale de services.

Enfin, en ce qui concerne l'équilibre de la balance des paiements, les règles de bouclage retenues ici sont les suivantes : l'équilibre de la balance des paiements relative au RdM hors UE est assuré par le taux de change réel tandis que le besoin de financement de la nation vis-à-vis des agents du reste de l'UE est la variable d'ajustement de la balance des paiements relative au reste de l'UE.

\subsection{La modélisation des instruments de politique agricole}

Dans un modèle d'EGC standard, les prix et les quantités s'ajustent simultanément pour assurer l'équilibre sur les marchés de tous les produits et de tous les facteurs primaires de production. Ce mode de fonctionnement des marchés ne tient pas compte de l'intervention publique qui peut introduire des contraintes sur les prix et/ou sur les quantités. Dans le modèle MEGAAF, un soin particulier a été apporté à la représentation des nombreux 
instruments de la PAC : système d'intervention, droits de douane à l'importation, subventions aux exportations, aides couplées à la production, aides à l'écoulement des produits sur le marché intérieur, quotas de production, gel des terres, aides compensatoires, aides sur les terres gelées, ....

Il nous est apparu nécessaire en effet de modéliser explicitement les principaux instruments de politique agricole plutôt que d'utiliser, à l'instar de nombreux modèles d'EGC, un indicateur synthétique de soutien (tel que l'Equivalent Subvention au Producteur -ESP- par exemple). Les deux types de modélisation ne sont pas équivalentes et les travaux de Kilkenny et Robinson (1988) et de Whalley et Wigle (1990) ont clairement montré les limites liées à l'utilisation de mesures synthétiques du soutien dans un modèle d'EGC. En outre, l'analyse des diverses voies de réforme possibles de la PAC nécessite la définition de scénarios très élaborés de changements de politique, impliquant une représentation aussi précise que possible de l'intervention publique multi-formes sur les marchés agricoles communautaires.

Dans le cas des produits de grandes cultures, les instruments de l'OCM des cultures arables explicitement modélisés dans MEGAAF dans la situation initiale sont les suivants ${ }^{8}$ : i) droits de douane fixes ${ }^{9}$ visant à assurer la protection à l'entrée vis-à-vis des pays tiers non communautaires ; ii) prix d'intervention pour les différentes céréales impliquant l'octroi de restitutions variables à l'exportation sur pays tiers (i. e. non communautaires) ; iii) taxes de co-responsabilité céréalière existant en 1990 ; iv) subventions couplées à la production de graines oléagineuses et de protéagineux ; v) subventions à l'exportation de produits transformés des céréales sur pays tiers.

\section{Définition des scénarios "de référence" et "protection zéro"}

Pour illustrer l'importance du maintien de la préférence communautaire dans la perspective d'évolution de la PAC vers l'affaiblissement du rôle des systèmes de soutien par les prix au profit d'autres formes de soutien à caractère plus découplé, deux scénarios de réforme radicale de l'OCM des cultures arables sont envisagés. Ils considèrent tous deux une suppression de toutes les mesures de soutien interne en vigueur initialement dans le secteur français des COP: Toutefois, dans le scénario dit "de référence", les droits de douane appliqués aux importations de céréales et de protéagineux en provenance du RdM sont conservés, tandis que dans le scénario dit "protection zéro" ces droits de douane sont supprimés.

\footnotetext{
${ }^{8}$ Rappelons que MEGAAF est calibré sur les données de l'année 1990. La situation initiale des secteurs d'activité agricoles correspond par conséquent à une situation antérieure à la réforme de la PAC de mai 1992. Les instruments de politique agricole initialement pris en compte sont donc ceux de la PAC pré-réformée. Les instruments de la PAC réformée (ainsi que divers systèmes d'aides directes assises sur les différents facteurs primaires de production pour le secteur des COP, cf. Gohin et al., 1998b) sont toutefois eux aussi explicitement modélisés même s'ils ne sont pas activés ici.

${ }^{9}$ Bien qu'en 1990, les marchés communautaires des différentes céréales étaient protégés par un prix minimum fixe (le prix de seuil) impliquant des droits variables (les prélèvements variables) à l'importation en provenance des pays tiers, ce système de protection a été modélisé dans MEGAAF sous la forme de droits de douane fixes pour traduire, par anticipation, les équivalents tarifaires de l'AAUR. Soulignons par ailleurs que la protection tarifaire sur les graines oléagineuses est nulle.
} 


\subsection{Le scénario "de référence"}

Les hypothèses du scénario "de référence" sont résumées dans le tableau 2 ci-dessous, en différenciant les hypothèses "politiques" relatives aux instruments et les hypothèses "techniques" relevant du mode de fonctionnement du modèle.

Tableau 2. Les hypothèses du scénario "de référence"

i) Hypothèses "politiques"

- Suppression du régime de l'intervention pour les différentes céréales.

- Suppression des subventions à la production des graines oléagineuses et des protéagineux.

- Suppression des taxes céréalières de co-responsabilité existant en 1990.

- Suppression des subventions à l'exportation vers le RdM des produits transformés des céréales.

- Maintien de la protection tarifaire sur les céréales et les protéagineux.

ii) Hypothèses "techniques"

- Application des hypothèses "politiques" définies ci-dessus à l'échelle de l'UE.

- Diminution de la contribution du secteur institutionnel "gouvernement français" au financement du secteur institutionnel "FEOGA" (Fonds Européen d'Orientation et de Garantie Agricole) équivalente à la baisse des dépenses de ce dernier, dépenses calculées uniquement en faveur des secteurs et des biens agricoles et agro-alimentaires français.

La première des hypothèses "techniques" stipule que toutes les hypothèses "politiques" du scénario "de référence" sont appliquées au secteur des COP à la fois en France et dans les autres pays de l'UE. Cela signifie que, suite à l'application du scénario "de référence", les mécanismes d'ajustement observés au niveau du secteur français des COP s'appliquent également au secteur des cultures arables du reste de l'UE. L'impact de la suppression des mesures de soutien interne au secteur des COP dans le reste de l'UE se traduit alors, dans MEGAAF, au travers des demandes d'exportation de céréales et d'oléoprotéagineux adressées à la France par le reste de l'UE. Ces demandes d'exportation enregistrent en effet des variations de prix qui, par définition, sont équivalentes à celles observées au niveau des marchés français.

La première des hypothèses "politiques" signifie quant à elle que les prix d'intervention en vigueur dans l'OCM des céréales sont supprimés. Cette hypothèse se traduit de la façon suivante dans MEGAAF. Dans la situation initiale, les prix de marché français des céréales sont déterminés par les conditions d'offre (i.e. offre domestique et importations en provenance du reste de l'UE et du RdM) et de demande (i.e. demande domestique et exportations vers le reste de l'UE et le RdM), observées sur les marchés céréaliers, sous la contrainte que ces prix ne tombent pas en dessous des prix d'intervention. En d'autres termes, en situation de déficit d'offre, le régime d'intervention n'est pas actif et seules les conditions de marché déterminent les prix de marché intérieurs des céréales, qui sont alors, à l'équilibre, supérieurs aux prix d'intervention ${ }^{10}$. En revanche, en situation d'excédent d'offre, les prix de marché français des céréales sont égaux aux prix d'intervention. Dans ce cas, le régime d'intervention est actif et l'équilibre des marchés céréaliers est obtenu via le dégagement des surplus sur le marché du $\mathrm{RdM}$, au moyen de restitutions variables à l'exportation. 
Dans le scénario "de référence", les prix d'intervention et le système des restitutions à l'exportation sur le RdM sont supprimés. Il en résulte que les prix de marché français des céréales sont déterminés uniquement par les conditions d'offre et de demande observées sur les marchés céréaliers. On perçoit ici l'importance du maintien de la protection tarifaire qui, dans le scénario "de référence", continue d'assurer un certain degré d'étanchéité entre les marchés céréaliers français (et plus généralement communautaires) et le marché du RdM.

A cet égard, soulignons que le scénario "de référence" peut être considéré comme incompatible avec la stricte application des engagements en matière de tarification dans le domaine des céréales, négociés par l'UE dans le cadre de l'AAUR et de l'Accord de Blair House. En effet, si l'on interprète l'abandon du régime d'intervention pour les différentes céréales, non pas comme la suppression des prix d'intervention, mais comme leur fixation à zéro, alors le maintien d'un certain niveau de protection tarifaire dans l'UE (hors celui permis dans le cadre de la clause de sauvegarde) est incompatible avec la disposition stipulant que, pour chaque céréale, la somme du prix à l'importation et de l'équivalent tarifaire ne peut, en aucun cas, excéder $155 \%$ du prix d'intervention.

On perçoit bien là le risque porté par cette disposition au regard du maintien de la préférence communautaire dans le long terme, dans l'optique d'une évolution de la PAC dans le sens d'une réduction graduelle des prix de soutien en vigueur dans l'OCM des céréales. L'objet du scénario "protection zéro" est justement d'illustrer les effets de la suppression de toutes les mesures de soutien interne dans le secteur français des COP, dans le cas où les marchés français des céréales et des protéagineux ne bénéficient plus d'aucune protection tarifaire.

\subsection{Le scénario "protection zéro"}

Le scénario "protection zéro" reprend toutes les hypothèses "politiques" et "techniques" du scénario "de référence" (cf. tableau 2), excepté celle du maintien de la protection tarifaire sur les céréales et les protéagineux. La mise en oeuvre dans MEGAAF de cet abandon de la protection tarifaire est relativement difficile, en particulier en ce qui concerne les céréales, pour trois raisons essentielles : i) la modélisation de type Armington adoptée dans le modèle pour représenter les flux d'importation entre la France et le RdM ; ii) la nécessité de poser une hypothèse a priori sur la réponse des cours mondiaux à la suppression de la protection tarifaire française (et communautaire) ; iii) la prise en compte de l'ajustement de la demande d'exportation du reste de l'UE à la France, consécutivement à l'abandon de la protection tarifaire également appliqué dans le reste de l'UE.

\section{i) La nécessaire adaptation de la modélisation initiale du bloc des importations françaises de céréales}

Dans MEGAAF, les flux d'importation de tous les produits entre la France et les zones reste de l'UE et RdM sont représentés par le biais d'une modélisation de type Armington, à l'aide d'une fonction CES. Ce type de modélisation est clairement restrictive en ce qui concerne les importations françaises de céréales en provenance du RdM, dans le cadre de la mise en oeuvre du scénario "protection zéro".

La modélisation "à la Armington" fait l'hypothèse que les produits domestiques sont différents des produits importés, le degré de différenciation entre les biens des deux origines étant mesuré par le paramètre de substitution de la fonction CES. Or, une critique couramment émise à l'encontre de ce choix de modélisation est

\footnotetext{
${ }^{10}$ Notons que dans ce cas, le déficit d'offre sur les marchés céréaliers français se traduit, à l'équilibre, par l'absence
} d'exportations vers le RdM. 
qu'il conduit implicitement, en dehors de tout instrument de politique commerciale, à protéger le marché domestique vis-à-vis de la concurrence des importations étrangères, via cette différenciation des produits dont le rôle s'apparente à celui d'une barrière non tarifaire aux importations. Morke et Tarr (1995) ont souligné cette limite de la modélisation de type Armington au travers d'une fonction CES, en particulier dans le cas où la part des importations étrangères dans l'approvisionnement du marché intétieur considéré est initialement faible. Ils montrent en effet que dans ce cas, ce type de modélisation des flux d'échange a tendance à figer la structure d'approvisionnement du marché intérieur considéré par rapport à la situation initialement observée ${ }^{11}$.

Le tableau 3 ci-dessous suggère par conséquent que si, à l'instar des études existantes qui ont simulé la suppression de la préférence communautaire à l'aide de modèles d'EGC (Harrison et al., 1995; Burniaux et al., 1990, par exemple), la modélisation initiale du bloc des importations françaises de céréales est conservée dans MEGAAF, alors l'abandon de la protection tarifaire du scénario "protection zéro" n'aura que peu d'effets sur les marchés céréaliers français.

En effet, le tableau 3 montre que dans la situation initiale, le montant total des droits de douane prélevés sur les importations françaises de céréales en provenance du RdM est faible au regard de la valeur de ces importations. Ainsi, en 1990, le montant des droits prélevés sur le blé tendre s'élève à 2 millions de francs, ce qui représente $3 \%$ seulement de la valeur des importations françaises de blé tendre en provenance du RdM. Ce pourcentage est encore plus faible pour les autres catégories de céréales distinguées dans MEGAAF. Ces données observées suggèrent par conséquent qu'en 1990, la France importait du RdM essentiellement des céréales de qualités différentes de celles produites sur le territoire national, ces importations étant soumises à des prélèvements plus faibles que ceux correspondant à l'écart moyen entre les prix des céréales "de qualité française" en vigueur sur les marchés mondiaux et les prix de seuil. En d'autres termes, en 1990, les prix de seuil protégeaient parfaitement les marchés céréaliers français de la concurrence des céréales étrangères en ce sens que la France n'importait pratiquement pas de céréales de qualité similaire à sa production domestique en provenance du RdM.

En second lieu, le tableau 3 montre également que dans la situation initiale, les céréales offertes sur le marché français sont principalement des céréales produites en France, les importations en provenance de pays tiers représentant une très faible part de l'approvisionnement domestique $(0,36 \%$ pour le blé tendre par exemple). Dans ce cas, on est donc exactement dans la situation correspondant à l'exemple de Morke et Tarr (1995), i.e. que pour une valeur finie de l'élasticité de substitution entre les céréales importées du RdM et les céréales d'origine française dans la fonction CES, même si l'abandon de la protection tarifaire entraînait une baisse très importante des prix à l'importation des céréales étrangères par rapport aux prix des céréales domestiques, la part des importations en provenance du RdM dans l'approvisionnement des marchés céréaliers français augmenterait

\footnotetext{
"Leur argumentaire est le suivant. Si l'élasticité de substitution entre le produit domestique et le produit importé est égale à 2 , une baisse de $50 \%$ du prix relatif du produit importé par rapport à celui du produit domestique conduit à une augmentation de $100 \%$ de la part des importations étrangères dans l'approvisionnement du marché intérieur. Si cette part est initialement de $0,5 \%$, elle ne sera que de $1 \%$ dans la situation finale. Il en résulte que même une baisse très importante du prix relatif du produit importé ne permet pas aux importations étrangères de conquérir une part substantielle du marché intérieur.
} 
certes, mais resterait très faible (excepté peut-être pour le maïs grain dont la part des importations d'origine tiers dans l'approvisionnement du marché intérieur est initialement plus forte).

Tableau 3. Les échanges français de céréales en 1990 (en millions de francs 1990)

\begin{tabular}{|c|c|c|c|c|}
\hline & Blé tendre & Orge & Maïs grain & $\begin{array}{l}\text { Autres } \\
\text { céréales }\end{array}$ \\
\hline Importations françaises du RdM, évaluées au prix CAF (1) & 66 & 1 & 324 & 62 \\
\hline Montant total des droits de douanes prélevés (2) & 2 & 0 & 5 & 1 \\
\hline Valeur totale des importations $(3)=(1)+(2)$ & 68 & 1 & 329 & 63 \\
\hline Equivalent tarifaire calculé (2) / (3) & $2,94 \%$ & $0 \%$ & $1,52 \%$ & $1,58 \%$ \\
\hline Offre totale sur le marché français (4) & 18739 & 7350 & 6144 & 3667 \\
\hline $\begin{array}{l}\text { Part des importations tiers dans l'approvisionnement du marché } \\
\text { français }(3) /(4)\end{array}$ & $0,36 \%$ & $0,01 \%$ & $5,35 \%$ & $1,71 \%$ \\
\hline Exportations françaises vers le RdM, évaluées au prix FAB (5) & 6138 & 1141 & 966 & 376 \\
\hline Montant total des restitutions aux exportations ( 6 ) & 4896 & 1256 & 966 & 399 \\
\hline Valeur totale des exportations $(7)=(5)+(6)$ & 11124 & 2397 & 1932 & 775 \\
\hline Part des Restitutions dans la valeur totale des exportations (6) / (7) & $44,82 \%$ & $52,39 \%$ & $50,00 \%$ & $51,48 \%$ \\
\hline
\end{tabular}

Finalement, les données relatives à la structure initiale des flux d'importations françaises de céréales en provenance du RdM, rapportées dans le tableau 3, suggèrent que si l'on maintient la modélisation initiale du bloc des importations céréalières dans MEGAAF, l'abandon de la protection tarifaire du scénario "protection zéro" reviendra implicitement à ne supprimer que la protection qui était "tangible" en 1990, i.e. la protection vis-à-vis des céréales tiers de qualité différente des céréales d'origine française $\mathrm{e}^{12}$. Les droits de douane appliqués à ces importations tiers étant initialement faibles, de même que leur part dans l'approvisionnement total des marchés céréaliers français, l'abandon de la protection tarifaire vis-à-vis de ce seul type d'importation tiers ne produira finalement que peu d'effets sur les marchés des céréales en France.

En d'autres termes, les marchés céréaliers français continueront d'être artificiellement protégés de la concurrence des importations en provenance du $\mathrm{RdM}$, la modélisation initiale du bloc des importations céréalières françaises issues du RdM étant seulement capable de "reproduire" le flux d'importation initial (i.e. le flux d'importation de céréales de qualité "non française" qui du fait même de cette différence de qualité ne concurrence pas vraiment les marchés français), et incapable de représenter la création d'un flux d'importation de céréales tiers parfaitement substituables aux céréales d'origine française qui, lui concurrencerait vraiment les marchés français.

Pour remédier à ce problème, deux solutions sont envisageables. La première, plus simple et plus rapide à mettre en oeuvre, consiste à conserver la modélisation initiale des flux d'importation françaises de céréales en provenance du RdM, mais en augmentant de manière substantielle l'élasticité de substitution de la fonction CES lorsque le scénario "protection zéro" est appliqué. On traduit ainsi le fait que l'abandon de la protection tarifaire

${ }^{12} \mathrm{La}$ protection des marchés français vis-à-vis des céréales étrangères parfaitement substituables à leurs homologues d'origine française n'est en effet pas "tangible" dans la situation initiale puisqu'elle ne transparaît pas dans les données de l'année 1990 dans la mesure où les flux d'importation correspondants, i.e. les flux sur lesquels portaient les prélèvements variables résultant de l'application des prix de seuil, étaient quasiment nuls. 
est susceptible de permettre à des céréales tiers de "qualité française" de pénétrer les marchés céréaliers français, i.e. que l'application du scénario "protection zéro" devrait rendre les céréales offertes par le RdM à la France plus substituables aux céréales d'origine domestique que dans la situation initiale. Toutefois, en raison des parts initiales très faibles des importations issues du RdM dans l'approvisionnement des marchés céréaliers français (et des niveaux initiaux très bas des droits de douane correspondants), cette élasticité devrait être augmentée dans des proportions très importantes pour que MEGAAF soit capable de rendre compte d'un réel mouvement de pénétration des céréales tiers sur les marchés français qui, intuitivement, devrait résulter de la suppression de toute protection aux frontières françaises.

La seconde solution consiste à modifier la modélisation initiale du bloc des importations françaises de céréales en provenance du $\mathrm{RdM}$ en distinguant deux types de céréales : des céréales de qualité "non française", très peu substituables aux céréales d'origine française, correspondant aux flux d'importation observés en 1990, et des céréales de qualité "française", parfaitement substituables aux céréales domestiques, non importées en 1990 mais susceptibles de l'être en l'absence de protection aux frontières nationales.

Nous supposons donc qu'il existe deux types d'importation de céréales tiers : des céréales imparfaitement substituables aux céréales françaises, notées $M I_{i}$, et des céréales parfaitement substituables aux céréales françaises, notées $M S_{i}$. Le programme de minimisation du coût d'approvisionnement du marché intérieur de la céréale $i$, correspondant à la quantité $X C_{i}$, s'écrit :

$$
\min _{Y D_{i}, M I_{i}, M S_{i}} P D_{i} \cdot Y D_{i}+P M S_{i} \cdot M S_{i}+P M I_{i} \cdot M I_{i}
$$

sous la contrainte :

$$
X C_{i}=\alpha_{i} \cdot\left(\delta_{i} \cdot\left(Y D_{i}+M S_{i}\right)^{-\rho_{i}}+\left(1-\delta_{i}\right) \cdot M I_{i}^{-\rho_{i}}\right)^{\frac{-1}{\rho_{i}}}
$$

où $P D_{i}$ est le prix de la céréale $i$ d'origine domestique offerte sur le marché français, $P M S_{i}$ le prix sur le marché intérieur de la céréale $i$, parfaitement substituable à son homologue française, importée du RdM, $P M I_{i}$ le prix sur le marché intérieur de la céréale $i$, imparfaitement substituable à la céréale d'origine domestique, importée du RdM, et $\alpha_{i}, \delta_{i}, \rho_{i}$ les paramètres de la fonction CES.

A l'équilibre, les conditions suivantes doivent être vérifiées :

$$
\begin{aligned}
& M S_{i}=0 \text { et } Y D_{i}>0 \text { si } P M S_{i}>P D_{i} \\
& M S_{i} \geq 0 \text { et } Y D_{i} \geq 0 \text { si } P M S_{i}=P D_{i} ; \\
& M S_{i}>0 \text { et } Y D_{i}=0 \text { si } P M S_{i}<P D_{i}
\end{aligned}
$$

Ainsi, si le prix des importations tiers de céréales $i$ parfaitement substituables est strictement supérieur au prix à la production domestique de cette même céréale, alors les importations tiers de céréales $i$ parfaitement substituables sont nulles. Ce cas correspond à la situation initiale, dans laquelle le prix $P M S_{i}$ ne pouvait être 
inférieur au prix de seuil, nettement supérieur au prix à la production de la céréale correspondante en vigueur sur le marché français. En revanche, quand le prix des importations tiers de céréales $i$ parfaitement substituables diminue au niveau du prix à la production domestique, suite à une réduction de la protection aux frontières par exemple, alors le volume importé de ce type de céréales peut devenir positif. Enfin, quand le prix des importations tiers de céréales $i$ parfaitement substituables est strictement inférieur au prix à la production domestique de cette même céréale, alors le volume importé de céréales $i$ parfaitement substituables est strictement positif tandis que la production domestique de cette céréale restant sur le marché français devient nulle.

Cette modélisation, qualifiée de complémentaire, permet ainsi de rendre compte de manière endogène de changements de régime d'importation. Dans les données de référence, les prix des importations tiers de céréales parfaitement substituables sont fixés à des niveaux très élevés (correspondant aux niveaux des prix de seuil en vigueur en 1990) de sorte que les flux d'importation initiaux de ce type de céréales soient toujours nuls.

Dans le scénario "protection zéro", nous supposons que les droits de droits de douane en vigueur en 1990, sur les protéagineux et sur les céréales imparfaitement substituables en provenance du RdM, sont supprimés. De la même façon, la protection, via les prix de seuil, vis-à-vis des céréales tiers parfaitement substituables est abandonnée. Il en résulte que les importations tiers de ce type de céréales s'effectuent aux prix mondiaux correspondants. C'est là qu'apparaît la seconde difficulté de mise en oeuvre du scénario "protection zéro" dans MEGAAF.

ii) La nécessité de poser une hypothèse a priori sur la réponse des cours mondiaux céréaliers

La réponse des prix mondiaux à un accroissement de la demande française d'importation de céréales parfaitement substituables adressée au RdM ne peut pas être déterminée de manière endogène. En effet, dans la situation initiale ce type de céréales est uniquement exporté par la France. Par conséquent, seule la demande d'exportation de ce type de céréales du RdM adressée à la France a été initialement calibrée. La France étant par hypothèse un grand pays à l'exportation sur les marchés céréaliers du RdM, la demande d'exportation des diverses céréales du RdM adressée à la France permet effectivement de déterminer de manière endogène la variation du prix mondial de chaque céréale mais uniquement en réponse à un ajustement de l'offre d'exportation française sur ces marchés.

En revanche, l'offre d'importation de céréales parfaitement substituables du RdM adressée à la France qui permettrait de déterminer de manière endogène la réponse des prix mondiaux céréaliers à un ajustement du volume de ces céréales importé par la France n'existe pas initialement. Elle est en outre impossible à calibrer sur les données observées.

Il est donc nécessaire de faire une hypothèse a priori sur la réponse des prix mondiaux des céréales à l'application du scénario "protection zéro". Au cours de l'année de référence, la France est exportatrice nette de céréales sur le marché du RdM. Les prix mondiaux initiaux des diverses céréales peuvent alors être calculés, du côté des exportations, en référence à la part des restitutions variables dans la valeur totale des exportations françaises vers le $\mathrm{RdM}$ de chaque céréale. Le tableau 3 indique que pour toutes les céréales considérées dans MEGAAF, les prix mondiaux sont initialement inférieurs de 40 à $50 \%$ aux prix de marché domestiques 
correspondants. A partir de cette situation initiale, nous supposons que le scénario "protection zéro" induit une augmentation des cours mondiaux des céréales de sorte qu'ils deviennent inférieurs de $30 \%$ aux prix de marché domestiques correspondants. Ainsi, dans le cas du blé tendre par exemple, notre hypothèse revient à anticiper une hausse de $27 \%$ du prix mondial en raison de la mise en oeuvre du scénario "protection zéro"13.

iii) La prise en compte des effets de la suppression de la protection tarifaire dans le reste de l'UE

Comme dans le scénario "de référence", toutes les mesures politiques du scénario "protection zéro" sont appliquées à l'échelle communautaire. En particulier, la suppression de la protection tarifaire pour les céréales et les protéagineux s'applique également au reste de l'UE. Il en résulte que tous les mécanismes d'ajustement au niveau de la demande d'importation de céréales en direction du RdM, précédemment décrits dans le cas de la France, jouent de la même façon dans le reste de l'UE. En d'autres termes, la suppression de la protection aux frontières est en mesure de conduire les pays du reste de l'UE, tout comme la France, à aller s'approvisionner en céréales plutôt sur les marchés du RdM. Toutes choses égales par ailleurs, la suppression de la protection tarifaire dans le reste de l'UE devrait par conséquent contribuer à réduire la demande d'exportation de céréales du reste de l'UE adressée à la France, au profit de celle adressée au RdM ${ }^{14}$.

Là encore, une hypothèse a priori doit être posée quant à la réponse de la demande d'exportation de céréales du reste de l'UE adressée à la France à la suppression de la protection tarifaire dans le reste de l'UE. Nous supposons ici que l'abandon de la protection aux frontières du reste de l'UE se traduit par une baisse exogène de $20 \%$ de la demande d'exportation des autres pays membres de l'UE adressée aux céréales françaises.

\section{Les résultats de simulation : L'importance du maintien de la préférence communautaire au regard du futur des secteurs agricole et agro-alimentaire français}

Il n'est pas question de présenter ici en détails l'ensemble des résultats de simulations des deux scénarios retenus. En effet, dans ces deux scénarios, les changements de politique envisagés touchent d'abord le secteur d'activité des cultures arables, puis "l'onde de choc" se propage dans l'ensemble des secteurs d'activité et institutionnels de l'économie française, via les marchés de tous les produits et de tous les facteurs primaires de production. MEGAAF étant constitué d'un grand nombre de secteurs d'activité, d'institutions et de biens, l'analyse détaillée de la totalité des résultats de simulations serait beaucoup trop longue.

Rappelons en outre que notre objet n'est pas de procéder à une évaluation de politique mais, plus simplement, d'illustrer l'importance pour l'agriculture française du maintien de la préférence communautaire dans la perspective d'évolution future de la PAC. Nous nous contentons donc de donner quelques "coups de projecteur" sur les résultats de simulations les plus marquants au regard de cette question.

\footnotetext{
${ }^{13}$ Cette hypothèse peut être rapprochée du résultat de Burniaux et al. (1990) qui montrent, à l'aide du modèle WALRAS, que la libéralisation des politiques agricoles dans tous les pays de l'OCDE entraîne une augmentation du prix mondial du blé tendre de $24 \%$.

${ }^{14}$ Notons ici que cet effet de détournement de la demande du reste de l'UE de l'origine française vers l'origine RdM contribue également à tirer les cours mondiaux céréaliers à la hausse, et renvoie par conséquent à l'hypothèse posée sur la réponse des prix mondiaux des céréales à l'application du scénario "protection zéro".
} 


\subsection{Les effets des scénarios "de référence" et "protection zéro" sur les équilibres de marché des céréales}

Tableau 4. Impacts des scénarios "de référence" et "protection zéro" sur les équilibres de marché des céréales (volumes en millions de francs 1990, variations en pourcentages par rapport à la base)

\begin{tabular}{|c|c|c|c|c|c|c|}
\hline & \multicolumn{3}{|c|}{ Blé tendre } & \multicolumn{3}{|c|}{ Orge } \\
\hline Variables & $\begin{array}{l}\text { équilibre } \\
\text { initial }\end{array}$ & $\begin{array}{l}\text { Scénario de } \\
\text { référence }\end{array}$ & $\begin{array}{l}\text { Scénario } \\
\text { protection } \\
\text { zéro }\end{array}$ & $\begin{array}{l}\text { équilibre } \\
\text { initial }\end{array}$ & $\begin{array}{l}\text { Scénario de } \\
\text { référence }\end{array}$ & $\begin{array}{c}\text { Scénario } \\
\text { protection } \\
\text { zéro }\end{array}$ \\
\hline Production domestique $Y_{i}$ & 36381 & $\begin{array}{c}29430 \\
-19,11 \%\end{array}$ & $\begin{array}{c}18752 \\
-48,46 \%\end{array}$ & 11373 & $\begin{array}{c}10022 \\
-11,88 \%\end{array}$ & $\begin{array}{c}6145 \\
-45,97 \%\end{array}$ \\
\hline Prix à la production domestique $P_{i}$ & 1 & $\begin{array}{c}0,8150 \\
-18,50 \%\end{array}$ & $\begin{array}{c}0,7000 \\
-30 \%\end{array}$ & . & $\begin{array}{c}0,8789 \\
-12,11 \%\end{array}$ & $\begin{array}{c}0,7000 \\
-30 \%\end{array}$ \\
\hline Exportation vers l'UE $E_{i, U E}$ & 8404 & $\begin{aligned} & 9309 \\
+ & 10,77 \%\end{aligned}$ & $\begin{array}{c}8036 \\
-4,38 \%\end{array}$ & 2149 & $\begin{array}{c}2292 \\
+6,67 \%\end{array}$ & $\begin{array}{c}2055 \\
-4,38 \%\end{array}$ \\
\hline Exportation vers le RdM $E_{i, R d M}$ & 9306 & $\begin{array}{c}0 \\
-100 \%\end{array}$ & $\begin{array}{c}0 \\
-100 \%\end{array}$ & 1875 & $\begin{array}{c}0 \\
-100 \%\end{array}$ & $\begin{array}{c}0 \\
-100 \%\end{array}$ \\
\hline $\begin{array}{l}\text { Volume du bien produit et vendu sur le } \\
\text { marché français } Y D_{i}\end{array}$ & 18671 & $\begin{array}{r}20120 \\
+7,76 \%\end{array}$ & $\begin{array}{c}10716 \\
-42,61 \%\end{array}$ & 7349 & $\begin{aligned} & 7729 \\
+ & 5,18 \%\end{aligned}$ & $\begin{array}{c}1491 \\
-58,62 \%\end{array}$ \\
\hline $\begin{array}{l}\text { Importation imparfaitement } \\
\text { substituable du RdM } M I_{i, R d M}\end{array}$ & 68 & $\begin{array}{c}47 \\
-31,07 \%\end{array}$ & $\begin{array}{c}40 \\
-40,78 \%\end{array}$ & 1 & $\begin{array}{c}1 \\
-17,92 \%\end{array}$ & $\begin{array}{c}1 \\
-35,82 \%\end{array}$ \\
\hline $\begin{array}{l}\text { Importation parfaitement substituable } \\
\text { du RdM } M S_{i . R d M}\end{array}$ & 0 & 0 & 10300 & 0 & 0 & 4514 \\
\hline $\begin{array}{l}\text { Volume du bien disponible pour le } \\
\text { marché français } X C_{i}\end{array}$ & 18739 & $\begin{array}{c}20163 \\
+7,60 \%\end{array}$ & $\begin{aligned} & 21048 \\
+ & 12,32 \%\end{aligned}$ & 7350 & $\begin{array}{r}7730 \\
+5,17 \%\end{array}$ & $\begin{aligned} & 8604 \\
+ & 17,07 \%\end{aligned}$ \\
\hline $\begin{array}{l}\text { Prix du bien composite offert sur le } \\
\text { marché français } P C_{i}\end{array}$ & 1 & $\begin{array}{c}0,8158 \\
-18,42 \%\end{array}$ & $\begin{array}{c}0,7010 \\
-29,90 \%\end{array}$ & 1 & $\begin{array}{l}0,8789 \\
-12,11 \%\end{array}$ & $\begin{array}{c}0,7000 \\
-30 \%\end{array}$ \\
\hline $\begin{array}{l}\text { Consommation intermédiaire totale } \\
C I T_{i}\end{array}$ & 16787 & $\begin{array}{r}17766 \\
+5,83 \%\end{array}$ & $\begin{array}{r}18247 \\
+8,70 \%\end{array}$ & 6115 & $\begin{array}{c}6322 \\
+3,39 \%\end{array}$ & $\begin{aligned} & 6830 \\
+ & 11,70 \%\end{aligned}$ \\
\hline
\end{tabular}

\begin{tabular}{|c|c|c|c|c|c|c|}
\hline & \multicolumn{3}{|c|}{ Maïs grain } & \multicolumn{3}{|c|}{ Autres céréales } \\
\hline Variables & $\begin{array}{l}\text { équilibre } \\
\text { initial }\end{array}$ & $\begin{array}{l}\text { Scénario de } \\
\text { référence }\end{array}$ & $\begin{array}{l}\text { Scénario } \\
\text { protection } \\
\text { zéro }\end{array}$ & $\begin{array}{l}\text { équilibre } \\
\text { initial }\end{array}$ & $\begin{array}{l}\text { Scénario de } \\
\text { référence }\end{array}$ & $\begin{array}{c}\text { Scénario } \\
\text { protection } \\
\text { zéro }\end{array}$ \\
\hline Production domestique $Y_{i}$ & 15070 & $\begin{array}{l}13715 \\
-8,99 \%\end{array}$ & $\begin{array}{c}8216 \\
-45,48 \%\end{array}$ & 5709 & $\begin{array}{c}5099 \\
-10,68 \%\end{array}$ & $\begin{array}{c}2867 \\
-49,78 \%\end{array}$ \\
\hline Prix à la production domestique $P_{i}$ & 1 & $\begin{array}{l}0,9110 \\
-8,90 \%\end{array}$ & $\begin{array}{c}0,7000 \\
-30 \%\end{array}$ & 1 & $\begin{array}{l}0,9555 \\
-4,45 \%\end{array}$ & $\begin{array}{c}0,7000 \\
-30 \%\end{array}$ \\
\hline Exportation vers I'UE $E_{i, U E}$ & 7625 & $\begin{array}{c}7989 \\
+4,77 \%\end{array}$ & $\begin{array}{l}7291 \\
-4,38 \%\end{array}$ & 1439 & $\begin{array}{c}1472 \\
+2,30 \%\end{array}$ & $\begin{array}{c}1376 \\
-4,38 \%\end{array}$ \\
\hline Exportation vers le RdM $E_{i, R d M}$ & 1630 & $\begin{array}{c}0 \\
-100 \%\end{array}$ & $\begin{array}{c}0 \\
-100 \%\end{array}$ & 666 & $\begin{array}{c}0 \\
-100 \%\end{array}$ & $\begin{array}{c}0 \\
-100 \%\end{array}$ \\
\hline $\begin{array}{l}\text { Volume du bien produit et vendu sur le } \\
\text { marché français } Y D_{i}\end{array}$ & 5815 & $\begin{array}{c}5726 \\
-1,53 \%\end{array}$ & $\begin{array}{c}924 \\
-84,10 \%\end{array}$ & 3604 & $\begin{array}{c}3627 \\
+0,65 \%\end{array}$ & $\begin{array}{c}1491 \\
-58,62 \%\end{array}$ \\
\hline $\begin{array}{l}\text { Importation imparfaitement } \\
\text { substituable du RdM } M I_{i, R d M}\end{array}$ & 329 & $\begin{array}{c}279 \\
-15,18 \%\end{array}$ & $\begin{array}{c}197 \\
-39,98 \%\end{array}$ & 63 & $\begin{array}{c}59 \\
-6,88 \%\end{array}$ & $\begin{array}{c}47 \\
-25,36 \%\end{array}$ \\
\hline $\begin{array}{l}\text { Importation parfaitement substituable } \\
\text { du } \operatorname{RdM} M S_{i, R d M}\end{array}$ & 0 & 0 & 5025 & 0 & 0 & 3090 \\
\hline $\begin{array}{l}\text { Volume du bien disponible pour le } \\
\text { marché français } X C_{i}\end{array}$ & 6144 & $\begin{array}{c}6003 \\
-2,30 \%\end{array}$ & $\begin{array}{c}6123 \\
-0,34 \%\end{array}$ & 3667 & $\begin{aligned} & 3686 \\
+ & 0,51 \%\end{aligned}$ & $\begin{array}{c}4622 \\
+26,05 \%\end{array}$ \\
\hline $\begin{array}{l}\text { Prix du bien composite offert sur le } \\
\text { marché français } P C_{i}\end{array}$ & 1 & $\begin{array}{l}0,9158 \\
-8,42 \%\end{array}$ & $\begin{array}{l}0,7124 \\
-28,76 \%\end{array}$ & 1 & $\begin{array}{l}0,9563 \\
-4,37 \%\end{array}$ & $\begin{array}{l}0,7039 \\
-29,61 \%\end{array}$ \\
\hline $\begin{array}{l}\text { Consommation intermédiaire totale } \\
C I T_{i}\end{array}$ & 10048 & $\begin{array}{r}10274 \\
+2,25 \%\end{array}$ & $\begin{aligned} & 11635 \\
+ & 15,79 \%\end{aligned}$ & 2408 & $\begin{array}{c}2367 \\
-1,71 \%\end{array}$ & $\begin{array}{c}2823 \\
+17,25 \%\end{array}$ \\
\hline
\end{tabular}


La situation initiale est caractérisée par un régime d'intervention actif pour les céréales. Ainsi, l'équilibre des marchés céréaliers français est assuré via l'exportation des surplus sur le marché du RdM, les volumes exportés pouvant s'ajuster grâce aux restitutions variables à l'exportation. Dans les deux scénarios simulés, la suppression du régime d'intervention redonne au prix de chaque céréale sa fonction de variable d'ajustement entre l'offre et la demande. Les prix à la production domestique obtenus à l'issue de l'application des deux scénarios sont donc des prix de marché qui équilibrent l'offre domestique et les différents usages de cette production domestique, i.e. les exportations vers le reste de l'UE, les exportations vers le RdM hors UE et les ventes sur le marché intérieur français.

\section{i) Les mécanismes d'ajustement à l'oeuvre dans le scénario "de référence"}

Dans le scénario "de référence", la suppression à l'échelle communautaire des mesures de soutien interne dans le secteur des COP entraine une diminution des prix à la production des quatre céréales, la baisse la plus importante étant enregistrée par le blé tendre (-18,50\% par rapport à la base), suivi de l'orge (-12,11\%), du maïs grain $(-8,90 \%)$ et enfin des autres céréales $(-4,45 \%)$. Cette baisse des prix à la production s'accompagne d'une réduction des niveaux de production domestique de chaque céréale : $-19,11 \%$ pour le blé tendre, $-11,88 \%$ pour l'orge, $-8,99 \%$ pour le maïs grain et $-10,68 \%$ pour les autres céréales, toujours par rapport à la base.

Parallèlement, du côté de la demande, les volumes de céréales utilisées en tant que consommation intermédiaire augmentent sous le double jeu d'un effet prix positif lié à la baisse des prix des céréales dans les différents usages domestiques (effet substitution lié à la baisse du prix propre) et d'un effet volume positif lié à l'accroissement de la production de biens dans les différents secteurs d'activité qui utilisent les céréales comme input intermédiaire, en particulier les secteurs agricoles animaux et le secteur agro-alimentaire de l'alimentation animale. Les différentiels de baisse de prix entre les quatre céréales expliquent que la demande dérivée de blé tendre augmente proportionnellement le plus ( $+5,83 \%$ par rapport à la base), suivie de celle de l'orge $(+3,39 \%)$ et de celle du maïs grain $(+2,25 \%)$. Dans le cas de l'agrégat des autres céréales, l'effet de la baisse des prix du blé, de l'orge et du maïs, effet prix croisé qui fait diminuer la demande dérivée d'autres céréales, toutes choses égales par ailleurs, "surcompense" l'effet positif de la baisse du prix propre et l'effet positif d'expansion des secteurs agricoles animaux et du secteur agro-alimentaire de l'alimentation animale. Il en résulte que la demande dérivée de l'agrégat autres céréales diminue de $-1,71 \%$ par rapport à la base.

Les mesures de soutien interne étant également supprimées dans le secteur des COP du reste de l'UE, les mêmes mécanismes que ceux décrits ci-dessus dans le cas de la France, jouent au niveau de l'offre et de la demande céréalières du reste de l'UE. Ils se traduisent par un accroissement de la demande des quatre céréales adressée à la France par le reste de l'UE. Ainsi, le scénario "de référence" conduit à un accroissement des exportations françaises de blé tendre $(+10,77 \%)$, d'orge $(+6,67 \%)$, de maïs grain $(+4,77 \%)$ et d'autres céréales $(+2,30 \%)$ vers le reste de l'UE.

En revanche, dans la situation finale, les céréales françaises ne sont pas compétitives sur le marché du RdM, si bien que les volumes exportés vers cette destination s'annulent. En effet, en dépit des baisses de prix de marché domestiques enregistrées par les quatre céréales et de l'ajustement à la hausse de leurs cours mondiaux $(+10 \%$ 
par rapport à la base), la situation finale est caractérisée par la persistance d'écarts entre les prix intérieurs et les prix mondiaux des quatre céréales. Or, le scénario "de référence" implique la suppression des restitutions à l'exportation sur pays tiers. Il en résulte que ces écarts ne peuvent plus être comblés via les restitutions, pour restaurer la compétitivité des céréales françaises sur le marché du RdM, comme c'était le cas dans la situation initiale.

A ce stade, le rôle du maintien de la protection tarifaire vis-à-vis des importations en provenance du RdM mérite d'être souligné. En effet, la persistance d'écarts entre les prix intérieurs et les cours mondiaux des céréales, observée à l'équilibre final, traduit le fait qu'en dépit de la suppression du régime d'intervention, les marchés céréaliers français (et plus généralement communautaires) continuent d'être "isolés" des marchés mondiaux. Plus précisément, l'équilibre en prix (à la production) des marchés français et communautaires continue d'être réalisé "indépendamment", en quelque sorte, de celui des marchés mondiaux. Cet "isolement" des marchés céréaliers français n'est possible que dans le cas où la protection tarifaire continue d'assurer la non compétitivité des céréales tiers sur les marchés domestiques.

Ainsi, le tableau 4 montre bien que dans le scénario "de référence", malgré des prix mondiaux des quatre céréales inférieurs à leurs prix intérieurs, les importations françaises de céréales parfaitement substituables aux céréales domestiques en provenance $\mathrm{du} \operatorname{RdM}$ sont toujours nulles ${ }^{15}$. Ce résultat découle directement de l'hypothèse de maintien de la protection tarifaire.

\section{ii) L'impact de la suppression de la protection tarifaire dans le cadre du scénario "protection zéro"}

Le tableau 4 indique que lorsque la protection tarifaire est abandonnée, i.e. lorsque la préférence communautaire n'est plus assurée, les marchés céréaliers français (et plus généralement communautaires) ne sont plus "isolés" des marchés mondiaux. Il en résulte que dans le scénario "protection zéro", les prix d'équilibre qui s'établissent sur les marchés français des quatre céréales sont égaux aux prix mondiaux. Ces derniers étant par hypothèse inférieurs de $30 \%$ aux prix intérieurs à l'issue de l'application du scénario "protection zéro" (cf. section 3), les prix à la production des quatre céréales diminuent de $-30 \%$ par rapport à la base dans ce scénario.

Les baisses de prix intérieurs étant plus fortes que celles observées dans le scénario "de référence", les effets du scénario "protection zéro" sur les équilibres de marché des quatre céréales sont nettement plus marqués. Ainsi, les niveaux de production domestique diminuent plus fortement : $-48,46 \%$ (contre $-19,11 \%$ dans le scénario "de référence") pour le blé tendre, $-45,97 \%$ (contre $-11,88 \%$ ) pour l'orge, $-45,48 \%$ (contre $-8,99 \%$ ) pour le maïs grain et $-49,78 \%$ (contre $-10,68 \%$ ) pour les autres céréales, par rapport à la base. De la même façon, l'ajustement à la hausse de la demande dérivée des quatre céréales est beaucoup plus important dans le scénario "protection zéro" que dans le scénario "de référence" : $+8,70 \%$ (contre $+5,83 \%$ ) pour le blé tendre, $+11,70 \%$ (contre $+3,39 \%$ ) pour l'orge, $+15,79 \%$ (contre $+2,25 \%$ ) pour le maïs grain et $+17,25 \%$ (contre $-1,71 \%$ ) pour les autres céréales, par rapport à la base.

\footnotetext{
${ }^{15}$ Les importations françaises de céréales tiers imparfaitement substituables aux céréales domestiques diminuent quant à elles sous l'effet de la baisse des prix intérieurs qui améliore la compétitivité des quatre céréales d'origine domestique relativement à celles en provenance du $\mathrm{RdM}$.
} 
Comme dans le scénario précédent, les mêmes mécanismes d'ajustement jouent dans le reste de l'UE, et ont tendance à faire croître la demande d'exportation de céréales du reste de l'UE. Toutefois, dans le scénario "protection zéro", la suppression de la préférence communautaire restaure la compétitivité des céréales originaires du RdM sur les marchés du reste de l'UE. Si bien que le reste de l'UE peut à présent s'approvisionner en céréales auprès du RdM plutôt que de s'adresser à la France. Cet effet se traduit par une baisse exogène (par hypothèse de $20 \%$, cf. section 3) de la demande d'exportation de céréales du reste de l'UE adressée à la France. Il en résulte que dans le scénario "protection zéro", les exportations françaises vers le reste de l'UE de chacune des quatre céréales diminuent de $-4,38 \%$ par rapport à la base.

La production domestique de céréales ayant fortement baissé, les exportations françaises vers le RdM sont, comme dans le scénario précédent, nulles tandis que les ventes de céréales domestiques sur le marché français diminuent fortement par rapport à la base $(-42,61 \%$ pour le blé tendre, $-58,62 \%$ pour l'orge, $-84,10 \%$ pour le maïs grain et $-58,62 \%$ pour les autres céréales).

La demande domestique de céréales augmentant, le déficit d'offre de céréales françaises est compensé par des importations de céréales parfaitement substituables en provenance du RdM. Ainsi, le tableau 4 montre que les importations françaises de ce type de céréales augmentent de manière très substantielle dans le scénario "protection zéro"16. Elles s'établissent, à l'équilibre final à 10,3 milliards de francs 1990 pour le blé tendre, 4,5 milliards de francs 1990 pour l'orge, 5 milliards de francs 1990 pour le maïs grain et 3,1 milliards de francs 1990 pour les autres céréales. Il en résulte que la structure d'approvisionnement des marchés céréaliers français est fortement modifiée à l'issue de l'application du scénario "protection zéro", relativement à la base et au scénario "de référence". Alors que dans la situation initiale et dans le scénario "de référence", les marchés français des quatre céréales sont approvisionnés en quasi-totalité (i.e. excepté le segment très limité des céréales de qualité non française) par des produits d'origine nationale, dans le scénario "protection zéro" les céréales non communautaires couvrent en moyenne près de $57 \%$ de l'approvisionnement des marchés français ( $48,9 \%$ pour le blé tendre, $52,5 \%$ pour l'orge, $82,1 \%$ pour le maïs grain et $66,9 \%$ pour les autres céréales).

En d'autres termes, l'abandon de la préférence communautaire rend les marchés céréaliers français beaucoup plus dépendants des importations de céréales étrangères. On peut noter en particulier, qu'à l'issue de l'application du scénario "protection zéro", la France devient presque totalement dépendante des importations en provenance du RdM pour le maïs grain.

\subsection{Les effets des scénarios "de référence" et "protection zéro" sur le secteur d'activité des grandes cultures}

De manière générale, la contraction de l'activité du secteur des grandes cultures $\mathrm{COP}$, consécutive à l'application des deux scénarios, se traduit par une réduction des volumes de facteurs de production primaires utilisés dans ce secteur, par rapport à la situation initiale. La contraction de l'activité étant plus marquée dans le scénario "protection zéro" que dans le scénario "de référence", la baisse des demandes dérivées de facteurs de production

\footnotetext{
${ }^{16}$ Tandis que, comme dans le cas du scénario "de référence" et pour les mêmes raisons, les importations françaises de céréales tiers imparfaitement substituables aux céréales domestiques diminuent par rapport à la base.
} 
dans le secteur des COP est plus forte lorsque la suppression des mesures de soutien interne s'accompagne de l'abandon de la préférence communautaire.

Sans entrer dans le détail, soulignons simplement que la mise en oeuvre du scénario "de référence" entraîne une diminution de la terre utilisée dans le secteur d'activité des grandes cultures de $-5,85 \%$ par rapport à la base tandis que la rémunération de ce facteur dans ce secteur d'activité décroît de $-56,98 \%$. Ce dernier chiffre reflète la capitalisation du soutien au secteur des grandes cultures, tel qu'il était accordé dans le cadre de la PAC préréformée, dans le prix de la terre. Dans le scénario "protection zéro", la demande dérivée de terre ainsi que la rémunération de ce facteur dans le secteur des COP diminuent plus fortement : $-17,26 \%$ et $-89,06 \%$ respectivement par rapport à la base. Bien évidemment, dans les deux scénarios, les terres libérées par le secteur des grandes cultures COP sont réallouées ${ }^{17}$ au profit des autres secteurs d'activité agricoles utilisateurs de terre (i.e., les secteurs de l'élevage utilisant des surfaces fourragères et les secteurs des vins et des autres produits de culture).

Au total, l'application des deux scénarios entraîne une baisse substantielle de la valeur ajoutée au coût des facteurs, i.e. nette des subventions d'exploitation et des taxes à la production, dégagée par le secteur des grandes cultures COP. Ainsi, cette valeur ajoutée diminue de $-16,966$ milliards de francs 1990 (soit -33,49\% par rapport à la base) dans le scénario "de référence" et de $-32,900$ milliards de francs 1990 (soit $-64,95 \%$ par rapport à la base) dans le scénario "protection zéro". Ces deux chiffres suggèrent que la suppression des mesures de soutien interne sans aucune compensation dans le secteur des cultures arables semble difficilement envisageable politiquement et socialement, au moins sur le court terme. Le dernier chiffre révèle quant à lui clairement l'enjeu d'un abandon de la préférence communautaire pour le secteur d'activité français des grandes cultures.

\subsection{Les effets des scénarios "de référence" et "protection zéro" sur les autres secteurs d'activité et sur le} bien-être économique global

Il n'est pas question ici d'entrer dans le détail des résultats de simulations relatifs à l'ensemble des autres secteurs d'activité pris en compte dans MEGAAF. Soulignons simplement que l'essentiel des effets des scénarios "de référence" et "protection zéro" sur les secteurs autres que celui des grandes cultures passe par i) la baisse des prix intérieurs des céréales, ii) l'ajustement des prix des facteurs primaires de production (travail et capital pour tous les secteurs d'activité, et terre pour les autres secteurs agricoles), iii) la variation du revenu disponible des ménages qui possèdent ces facteurs de production et iv) les bouclages macro-économiques, en particulier via les économies réalisées par le gouvernement français sur son transfert au FEOGA (du fait de la suppression des mesures de soutien interne dans le secteur des $\mathrm{COP}$ ) qui le conduisent à accroître sa consommation de services (cette variable étant, dans le cadre des règles de bouclage adoptées ici, la variable d'ajustement de l'équilibre du budget du gouvernement français).

Parmi l'ensemble des secteurs d'activité les plus "exposés" à la baisse des prix des céréales, ceux de l'alimentation animale et de l'élevage constituent un maillon important du phénomène de propagation des effets

\footnotetext{
${ }^{17} \mathrm{Ce}$ mouvement de réallocation de la terre au détriment du secteur des COP et en faveur des autres secteurs d'activité agricoles étant nettement plus ample dans le scénario "protection zéro" que dans le scénario "de référence".
} 
des deux scénarios simulés dans l'ensemble de l'économie française. Les conséquences des scénarios "de référence" et "protection zéro" sur ces secteurs sont donc présentées rapidement, à titre d'exemple du mécanisme de propagation du choc initial dans le secteur des COP vers les autres secteurs d'activité d'une part, et pour donner une vision un peu plus large des effets des deux scénarios sur l'ensemble de l'agriculture française, d'autre part. Le tableau 5 ci-dessous rapporte les principaux effets des deux scénarios sur les secteurs d'activité de l'alimentation animale, de l'élevage et des viandes.

Tableau 5. Les principaux effets des scénarios "de référence" et "protection zéro" sur les secteurs d'activité de l'alimentation animale, de l'élevage et des viandes (variations en pourcentages par rapport à la base)

\begin{tabular}{|l|c|c|}
\hline Variables & Scénario de référence & Scénario protection zéro \\
\hline Aliments composés & & \\
Production domestique & $+2,74$ & $+6,69$ \\
Prix à la production & $-1,50$ & $-3,77$ \\
\hline Porc & & \\
Production d'animaux vivants & $+3,23$ & $+7,31$ \\
Prix à la production des animaux vivants & $-3,26$ & $-8,59$ \\
Production de viande & $+2,36$ & $+5,09$ \\
Prix à la production de la viande & $-2,06$ & $-4,31$ \\
Consommation finale de viande & $+1,78$ & $+3,86$ \\
\hline Volaille & & \\
Production d'animaux vivants & $+2,68$ & $+7,05$ \\
Prix à la production des animaux vivants & $-2,24$ & $-6,33$ \\
Production de viande & $+2,62$ & $+6,26$ \\
Prix à la production de la viande & $-1,76$ & $-3,97$ \\
Consommation finale de viande & $+1,42$ & $+3,37$ \\
\hline bovin & & \\
Production d'animaux vivants & $+7,06$ & $+16,23$ \\
Prix à la production des animaux vivants & $-1,44$ & $-3,52$ \\
Production de viande & $+6,22$ & $+13,73$ \\
Prix à la production de la viande & 0 & 0 \\
Consommation finale de viande & $-0,40$ & $-0,78$ \\
\hline
\end{tabular}

\section{i) Les principaux ajustements dans le secteur d'activité de l'alimentation animale}

Dans le secteur d'activité de l'alimentation animale, la baisse des prix des céréales consécutive à l'application du scénario "de référence" induit deux effets favorables à la consommation de céréales par ce secteur : un effet de substitution et un effet d'expansion. A production d'aliments composés inchangée, la baisse des prix des quatre céréales entraîne un effet substitution favorable aux céréales, au détriment des autres ingrédients (en particulier des ingrédients importés tels que le tourteau de soja et les produits substituts des céréales). A cet effet de substitution s'ajoute un effet d'expansion de l'activité du secteur de l'alimentation animale lié à la baisse du coût de fabrication des aliments composés. Cet effet d'expansion conduit le secteur de l'alimentation animale à accroître sa consommation de tous les ingrédients incorporés dans les rations pour animaux. Au total, l'application du scénario "de référence" se traduit par une augmentation de $+2,74 \%$ de la production domestique d'aliments composés et une diminution de $-1,50 \%$ du prix à la production correspondant.

Les mécanismes d'ajustement décrits ci-dessus dans le cas du scénario "de référence" s'appliquent également au scénario "protection zéro". Toutefois, la baisse des prix des céréales étant plus marquée dans ce second scénario, la production domestique d'aliments composés augmente plus fortement ( $+6,69 \%$ par rapport à la base) tandis que leur prix à la production enregistre une baisse plus importante $(-3,77 \%)$. 
A ce stade de l'analyse, il est important de noter que les baisses de prix substantielles des céréales qu'impliquent la mise en oeuvre des scénarios "de référence" et "protection zéro" ne se traduisent pourtant, in fine, que par une diminution très modeste du prix à la production (et à la consommation) des aliments composés. Ce résultat provient essentiellement du fait que les céréales (et plus généralement l'ensembles des ingrédients de l'alimentation animale) ne représentent qu'une partie seulement des coûts totaux en consommations intermédiaires pour l'industrie de l'alimentation animale.

ii) Les principaux ajustements dans les secteurs d'activité de l'élevage et des viandes

Les secteurs d'activité de l'élevage bénéficient quant à eux d'une baisse du coût de l'alimentation, par l'intermédiaire de la baisse du prix des céréales consommées en l'état et par le biais de la diminution du prix des aliments composés. Ainsi, le tableau 5 révèle que le scénario "de référence" conduit à une augmentation de la production domestique de porcs $(+3,23 \%$ par rapport à la base), de volailles $(+2,68 \%)$ et de bovins $(+7,06 \%)$ vivants $^{18}$. La production domestique de bovins augmente proportionnellement plus que celle de porcs et de volailles car même si l'ensemble de l'alimentation animale, fourrages exclus, représente une part plus élevée du coût de production dans le cas des monogastriques que dans celui des herbivores bovins, ces derniers bénéficient en plus d'une réallocation d'une partie des surfaces agricoles libérées par le secteur des COP.

A l'issue du scénario "de référence", les prix à la production des trois types d'animaux vivants sont plus faibles que dans la situation initiale : $-3,26 \%$ pour le porc, $-2,24 \%$ pour la volaille et $-1,44 \%$ pour les bovins. Cette baisse des prix à la production se répercute, pour partie au moins, au niveau des prix à la demande d'animaux vivants. Il en résultc que le secteur d'activité de l'abattage enregistre une diminution de ses coûts d'approvisionnement en animaux vivants. Ainsi, les productions domestiques des différentes viandes offertes par le secteur d'activité de l'abattage augmentent : $+2,36 \%$ pour la viande de porc, $+2,62 \%$ pour la viande de volaille et $+6,22 \%$ pour la viande bovine. Parallèlement les prix à la production des viandes porcine et avicole diminuent de $-2,06 \%$ et $-1,76 \%$ respectivement. En revanche, le prix à la production de la viande bovine est inchangé en raison du régime d'intervention appliqué à ce produit, régime qui permet de maintenir le prix à la production au niveau du prix d'intervention supposé inchangé ${ }^{19}$. Il en résulte que le prix relatif à la production de la viande bovine augmente par rapport à ceux des deux autres viandes, si bien que la production domestique de viande bovine augmente plus que proportionnellement par rapport à celle des deux autres viandes.

On retrouve l'effet inverse au niveau de la demande finale de viandes, le prix à la consommation finale de la viande bovine s'ajustant proportionnellement plus faiblement à la baisse que ceux des viandes de porc et de volaille. Ainsi, dans le scénario "de référence", la consommation finale de viande porcine s'accroît de $+1,78 \%$, celle de viande de volaille de $+1,42 \%$, tandis que celle de viande bovine diminue de $-0,40 \%$. On retrouve là l'un des effets pervers du régime d'intervention en vigueur dans l'OCM bovine qui, en cas de baisse des prix des

\footnotetext{
${ }^{18}$ La production domestique de lait à la ferme est quant à elle contrôlée par le quota à la production, supposé inchangé par rapport à la situation initiale.

${ }^{19}$ L'équilibre du marché de la viande bovine est alors assuré par le biais de l'augmentation des exportations sur pays tiers, au moyen de restitutions variables.
} 
céréales, induit une détérioration de la compétitivité prix de la viande bovine par rapport aux viandes porcine et avicole.

Bien évidemment, les mêmes mécanismes jouent dans le scénario "protection zéro", mais, là encore, les baisses de prix des quatre céréales étant nettement plus importantes, les effets sur les secteurs d'activité de l'alimentation animale, de l'élevage et des viandes sont beaucoup plus marqués. Dans ce second scénario, la production domestique de porcs vivants s'accroît de $+7,31 \%$ par rapport à la base, celle de volailles de $+7,05 \%$ et celle de bovins de $+16,23 \%$. Les baisses des prix à la production correspondants s'établissent à respectivement $-8,59 \%$, $-6,33 \%$ et $-3,52 \%$. La production domestique des trois types de viandes augmente également dans des proportions plus importantes que dans le scénario précédent : $+5,09 \%$ pour la viande porcine, $+6,26 \%$ pour la viande avicole et $+13,73 \%$ pour la viande bovine. Les prix à la production des viandes de porc et de volaille enregistrent des baisses plus marquées que dans le scénario "de référence" ( $-4,31 \%$ et $-3,97 \%$ respectivement) tandis que celui de la viande bovine est toujours inchangé par rapport à la situation initiale en raison du régime d'intervention en vigueur. Enfin, à l'instar du scénario précédent, la consommation finale de viandes de porc et de volaille augmente, $+3,86 \%$ et $+3,37 \%$ respectivement, tandis que celle de viande bovine diminue de $0,78 \%$.

\section{iii) Les effets des deux scénarios en termes de bien-être économique global}

A l'issue de l'ensemble des ajustements provoqués par la mise en oeuvre des scénarios "de référence" et "protection zéro" au sein de l'économie française, le bien-être économique global national, mesuré par la variation équivalente des secteurs institutionnels, augmente de $+3,229$ milliards de francs 1990 dans le premier scénario et de seulement $+0,833$ milliard de francs 1990 dans le second. Ce résultat, a priori contre intuitif, est une illustration de la théorie du second rang. En effet, dans un monde de second rang caractérisé par de nombreuses imperfections et distorsions sur les marchés, supprimer une de ces distorsions (la protection tarifaire vis-à-vis des importations de céréales et de protéagineux en provenance des pays non communautaires dans notre cas) ne garantit pas une amélioration du bien-être économique global (voir Schubert et Letournel, 1991 ; Harrison et al., 1995, par exemple).

\section{Eléments de conclusion}

La préférence communautaire est un principe fondamental de la PAC, dont l'objet est de protéger les marchés agricoles intérieurs de l'UE de la concurrence des importations en provenance des pays tiers. Si l'AAUR ne remet pas en cause le maintien de la préférence communautaire à court terme, il constitue un pas décisif dans la voie de l'abandon du principe même de le préférence communautaire à plus long terme.

Cette contrainte particulière issue de l'AAUR, et qui sera vraisemblablement renforcée lors des prochains cycles de négociations multilatérales dans le cadre de l'OMC, a été largement négligée dans les débats sur les perspectives d'évolution de la PAC. Pourtant, ce papier montre que si la PAC continue d'évoluer dans la voie ouverte par la réforme de mai 1992, i.e. dans le sens de l'affaiblissement du rôle des systèmes de soutien par les prix au profit d'autres formes de soutien à caractère plus découplé, le maintien de la préférence communautaire pourrait se révéler un élément clé au regard du futur des secteurs agricoles et agro-alimentaires français, et plus généralement communautaires. 
Les deux scénarios de réforme radicale de l'OCM des céréales et des oléoprotéagineux réalisés à l'aide de MEGAAF montrent en effet que la suppression des mesures de soutien interne dans le secteur des COP induit un choc en termes de contraction de l'activité de ce secteur beaucoup plus limité lorsque la préférence communautaire est maintenue que lorsque cette dernière est simultanément supprimée. Les résultats de simulations suggèrent en outre clairement que l'abandon de la préférence communautaire porte le risque de remettre en cause l'indépendance de la France (et plus généralement de l'UE) vis-à-vis des importations céréalières étrangères.

L'originalité de notre analyse tient essentiellement à l'adaptation de la modélisation du bloc des importations céréalières françaises qui, en distinguant deux types de céréales étrangères (imparfaitement et parfaitement substituables aux céréales d'origine nationale), permet de représenter de manière endogène un changement de régime d'importation. Une telle modélisation est susceptible de rendre compte d'un véritable mouvement de pénétration des céréales étrangères sur les marchés français, suite à la suppression de la protection aux frontières. La modélisation usuelle de type Armington, généralement adoptée dans les modèles d'EGC, ne permet pas de représenter un tel mouvement car, même en dehors de tout instrument de politique commerciale, elle érige des barrières artificielles à l'importation et tend ainsi à figer la structure d'approvisionnement des marchés intérieurs par rapport à la situation initiale. Ceci explique en grande partie pourquoi les études existantes proposant des scénarios de réforme de la PAC, dont l'abandon de la préférence communautaire fait partie intégrante, simulés à l'aide de modèles d'EGC, pour la plupart à modélisation Armington usuelle, conduisent à des résultats qui semblent beaucoup moins "dangereux" pour le secteur agricole communautaire que ne le suggèrent les résultats de cette étude.

Il est important de souligner une fois encore que les résultats obtenus ici sont contingents aux hypothèses posées, en particulier à celle adoptée sur la réponse des cours mondiaux des céréales à la suppression de la préférence communautaire dans le secteur des COP. Il est également bien évident que les scénarios de réforme de l'OCM des cultures arables envisagés dans cette étude relève d'une vision très partielle des perspectives d'évolution à long terme de la PAC dans son ensemble. En outre, il est clair que si les résultats obtenus sont susceptibles d'illustrer et d'attirer l'attention sur l'importance du maintien de la préférence communautaire, ils ne permettent en aucun cas de saisir la mesure globale des enjeux de la remise en cause de ce principe fondamental de la PAC pour l'ensemble de la sphère agricole et agro-alimentaire française et communautaire.

Les recherches futures devront par conséquent s'efforcer d'élargir la présente analyse, limitée au seul secteur des $\mathrm{COP}$, pour la replacer dans une vision plus globale de l'évolution de la PAC dans son ensemble. 


\section{Références}

Bureau J.C., Gohin A., Guyomard H., Le Mouël C., 1996, CAP response to the 1996 US FAIR Act. Paper prepared for the one-day conference organised by the Agricultural Economics Society on "The US Farm Bill : Implications for further CAP reform", London, November.

Burniaux J.M., Martin J.P., Delorme F., Lienert I., Van der Mensbrugghe D., 1990, Economy-wide effects of agricultural policies in OECD countries : a GE approach using the WALRAS model. In Goldin I. and Knudsen O., eds, Agricultural Trade Liberalization : Implications for Developing Countries, OECD, Paris.

De Melo J., Tarr D., 1992, A General Equilibrium Analysis of Foreign Trade Policy. The MIT Press, Cambridge, Massachusetts.

Gohin A., 1998, Modélisation du complexe agro-alimentaire français dans un cadre d'équilibre général. Document de travail MEGAAF n ${ }^{\circ}$ 6, Equipe PAM, Unité INRA-ESR, Rennes.

Gohin A., Guyomard H., Le Mouël C., 1998a, Eléments de définition d'une Politique Agricole Commune pour le XXIème siécle. Economie Rurale, n²44, pp.22-31.

Gohin A., Guyomard H., Le Mouël C., 1998b, Conséquences de modifications des niveaux et/ou des modalités d'octroi du soutien interne dans le secteur français des grandes cultures, céréales et oléoprotéagineux : analyse en équilibre général. Rapport intermédiaire pour le Ministère de l'Agriculture, de la pêche et de l'Alimentation, DAFE, INRA-ESR, Rennes.

Guyomard H., Mahé L.P., 1995a, Le GATT et la nouvelle Politique Agricole Commune : une réforme inachevée. Revue Economique, n 46, pp. 657-666.

Guyomard H., Mahé L. P., 1995b, La nouvelle instrumentation de la Politique Agricole Commune. Economie et Prévision, $\mathrm{n}^{\circ} 117-118$, pp. 15-30.

Guyomard H., Baudry M., Carpentier A., 1996, Estimating crop supply response in the presence of farm programmes : application to the CAP. European Review of Agricultural Economics, n²3, pp. 401-420.

Guyomard H., Herrard N., Le Mouël C., Le Roux Y., Trochet T., 1996b, L'Accord Agricole de l'Uruguay Round et la réforme de la Politique Agricole Commune : éléments d'analyse et de prospective. Rapport pour le Commissariat Général du Plan, ENSA-EGSS, INRA-ESR, Rennes.

Guyomard H., 1997, La réforme de l'Organisation Commune de Marché des grandes cultures (céréales, oléagineux, protéagineux) : quelques éléments de réflexion. Intervention au congrès de l'AGPM, Montpellier, 18 Septembre.

Harrison G.W., Rutherford T.F., Wooton I., 1995, Liberalizing agriculture in the European Union. Journal of Policy Modeling, vol. 17, pp. 223-256.

Helmar M. D., Meyers W. H., Hayes D. J., 1994, GATT and CAP Reform : Different, Similar or Redundant. In Anania, Carter and McCalla (eds), Agriculture Trade Conflicts and GATT: New Dimensions in US-European Agricultural Trade Relations, Westview Press.

Josling T., 1993, The Reformed CAP and the Industrial World. VIIth EAAE Congress, Stresa, Italy.

Josling T, Tangermann S., 1992, Mac-Sharry or Dunkel : Which Plan Reforms the CAP ? IATRC Working paper 92-10.

Kilkenny M., Robinson S., 1988, Modelling the removal of production incentive distortions in the US agricultural sector. XX International Conference of Agricultural Economists, Buenos Aires, Argentina, August 27-September 1. 
Morke M., Tarr D., 1995, Reforming Hungarian agricultural trade policy: a quantitative evaluation. Welwirtschaftliches Archiv, Review of World Economics, vol. 131, pp. 106-131.

Robinson S., Kilkenny M., Hanson K., 1990, The USDA/ERS Computable General Equilibrium (CGE) Model of the United States. United States Department of Agriculture, Economic Research Service, Agricultural and Rural Economy Division, Staff Report nAGES 9049.

Shoven J.B., Whalley J., 1984, Applied General Equilibrium Models of Taxation and International Trade : An Introductory Survey. Journal of Economic Literature, vol. 22, pp. 1007-1051.

Schubert K., Letournel P.Y., 1991, Un modèle d'équilibre général appliqué à l'étude de la fiscalité française : résultats de long terme. Economie et Prévision, n 98, pp. 83-101.

USDA, 1994, Effects of the Uruguay Round Agreement on US Agricultural Commodities. USDA, Office of Economics, Economic Research Service, GATT-1.

Whalley J., Wigle R., 1990, Terms of trade effects, agricultural trade liberalization and developing countries. In Goldin I. and Knudsen O., eds, Agricultural Trade Liberalization: Implications for Developing Countries, OECD, Paris. 